\title{
论文
}

\section{基于铝合金板材应力反求与应力评测的飞机 结构件加工变形控制新方法}

秦国华 ${ }^{1}$, 王华敏 ${ }^{2 *}$, 林锋 ${ }^{1}$, 孙士平 ${ }^{1}$, 郭翊翔 ${ }^{1}$, 吴铁军 ${ }^{3}$, 吴竹溪 ${ }^{1}$

1. 南昌航空大学航空制造工程学院, 南昌 330063;

2. 南京航空航天大学机电学院, 南京 210016 ;

3. 东莞职业技术学院机电工程系, 东莞 523808

*E-mail: 33008@nchu.edu.cn

收稿日期：2019-03-06; 接受日期：2019-05-27; 网络版发表日期：2019-10-08 国家自然科学基金(批准号: 51765047, 51465045)、江西省主要学科学术与技术带头人资助计划(批准号: 20172BCB22013)和江西省重大研发 计划(批准号: 20192BBEL50001)资助项目

摘要 在飞机结构件的高速切削过程中, 随着7075铝合金厚板的材料去除, 残余应力释放成为加工变形的关键因 素. 因此, 研究加工变形随毛坏初始残余应力释放的演化规律, 是进行加工质量控制的核心环节和基础, 对于实现 加工过程的高效化和精密化至关重要. 首先依据弯曲变形理论, 推导出工件随材料去除而产生的加工变形力学模 型, 通过厚向逐层施加单位应力, 利用有限元方法计算变形因子, 将力学模型分解为变形因子与残余应力之积的 数学形式. 其次, 将加工要求作为加工变形的控制限, 结合残余应力的自平衡特性建立残余应力规范模型, 采用夹 逼原则这一数学技巧, 通过引入单位变量将应力规范模型转化为齐次线性不等式组, 提出应力规范模型的枢轴变 换反求技术. 最后, 通过任意变量转化为两个非负变量之差的数学技巧, 提出了毛坏的实测应力值为应力规范的 解的线性规划判断方法, 实现了加工变形满足加工要求的预先评估. 通过枢轴变换和线性规划方法将被动的加工 变形分析转化为主动的残余应力评测, 为切削上游工艺提供合理的出厂应力标准, 实现毛坏制备与切削工艺的有 机结合.

关键词毛坏初始残余应力, 高速切削, 加工变形, 线性规划, 枢轴变换, 应力规范

\section{1 引言}

飞机整体结构件有着重量轻、装配时间短、生产 效率高和生产成本低的优势, 广泛应用于航空领域 ${ }^{[1]}$. 然而，由于尺寸大，且在加工过程中材料去除率高达 $90 \%$ 以上, 造成飞机结构件刚度较低, 毛坏初始残余应 力的存在容易引发加工变形 ${ }^{[2,3]}$. 虽然通过预拉伸工艺
可以大幅度地降低铝厚板中的残余应力水平 ${ }^{[4]}$ ，但是 残留部分的残余应力仍能引发变形问题.

Liu等人 ${ }^{[5]}$ 在通过层削法获得 7085-T7452铝合金 锻件初始残余应力的基础上, 利用ANSYS有限元软件 进行了挡风玻璃框架结构件变形分析, 经实验测试后 认为, 有限元方法能够有效地预测初始残余应力引起 的加工变形. Zhang等人 ${ }^{[6]}$ 使用轮廓法获得 7050-T7451

引用格式: 秦国华, 王华敏, 林锋, 等. 基于铝合金板材应力反求与应力评测的飞机结构件加工变形控制新方法. 中国科学: 技术科学, 2020, 50: 85-102 Qin G H, Wang H M, Lin F, et al. A new approach to deformation control of aeronautical monolithic components of aluminum alloy plates based on stress inverse and stress evaluation (in Chinese). Sci Sin Tech, 2020, 50: 85-102, doi: 10.1360/SST-2019-0089 
铝厚板的初始残余应力，通过计算出不同位置处的 $\mathrm{T}$ 形件加工变形, 认为零件在适当的厚度位置可以有效 地降低加工变形. Cerutti等人 ${ }^{[7,8]}$ 以层剥法测量得到毛 坏中的初始残余应力, 通过在FORGE有限元软件中加 入布朗运算，实现了加工变形的分析与预测. Nervi等 人 $^{[9]}$ 利用开槽法测出初始残余应力后, 利用NavierLamè方程实现了 7050-T7451铝合金厚板加工变形的 计算和分析，指出零件的最终变形不仅受初始残余应 力的影响，而且还与零件在毛坏中的位置有关，但仅 能分析材料去除方式为简单厚板层削 ${ }^{[10]}$ 和开单槽 ${ }^{[11]}$ 的弯曲变形情况.

事实上，铝厚板制造企业注重的是材料力学性能， 而航空整体结构件数控加工厂关心的则是残余应力. 企业之间没有交汇点, 相互脱节, 导致关于加工变形的 研究，均局限在“将残余应力赋值给毛坏，采用单元生 死技术模拟材料去除方式，施加位移约束后进行计算” 这一闭环式的被动分析方法上. 由此造成的加工变形 问题依然存在，至今仍为数控加工企业难以解决的技 术瓶颈.

为此，本文重点考虑毛坏的初始残余应力水平和 分布，建立航空整体结构件铣削成形过程中的加工变 形力学模型，再以加工要求为控制限，通过单位力法 获得关于残余应力厚向分布的齐次线性不等式，最后 构建齐次线性不等式的枢轴变换求解方法，将被动的 加工变形分析变为主动的残余应力评测，为切削上游 工艺提供合理的应力指南，实现毛坏初始残余应力和 切削工艺的有机结合.

\section{2 加工变形分析}

在高速切削过程中, 初始残余应力是加工变形的 关键因素. 毛坏制造过程中产生的初始残余应力，如 果分布不合理，即使通过后续的工艺优化也无法减小 零件变形. 为此, 制订合理的应力规范是控制零件最 终变形的最佳手段.

\section{1 残余应力释放}

毛坏(即铝合金厚板)在轧制方向 $X$ 上的尺寸为 $l$ $(\mathrm{mm})$, 横向方向 $Y$ 上的尺寸为 $w(\mathrm{~mm})$, 厚度方向 $Z$ 上的 尺寸为 $h(\mathrm{~mm})$. 板内的初始残余应力沿厚度方向 $Z$ 处于 平衡状态, 其应满足下列方程:

$$
\begin{aligned}
& \left\{\begin{array}{l}
\int_{M+W} \sigma_{X}(z) \mathrm{d} z=0, \\
\int_{M+W} \sigma_{X}(z) z \mathrm{~d} z=0,
\end{array}\right. \\
& \left\{\begin{array}{l}
\int_{M+W} \sigma_{Y}(z) \mathrm{d} z=0, \\
\int_{M+W} \sigma_{Y}(z) z \mathrm{~d} z=0,
\end{array}\right.
\end{aligned}
$$

其中, $\sigma_{X}(z)$ 为轧制方向 $X$ 的分力; $\sigma_{Y}(z)$ 为横向方向 $Y$ 的 分力.

毛坏经切削加工成形后，被区分为两个区域 $M$ 和 $W$. 区域 $M$ 表示从铝合金厚板上切除的多余材料, 而区 域 $W$ 表示材料切除后的成形零件, 如图1所示.

随着加工过程中材料 $M$ 的逐步去除, $M$ 内的残余 应力 $\sigma_{X}(z)$ 与 $\sigma_{Y}(z)$ 不断得到释放. 但是, 相比于材料的屈 服极限 $\sigma_{s}$, 残余应力 $\sigma_{X}(z)$ 和 $\sigma_{Y}(z)$ 的值较小, 故残余应力 的释放过程可以视为铝合金厚板的弹性变形过程. 由 此可见，残余应力释放过程可以认为材料是一步去除 到位的切削过程, 不需要考虑去除顺序. 为此, 可以将 区域 $M$ 和区域 $W$ 分开看待, 如图2所示. 这样, 随着材料 $M$ 的去除, 其相应内部的残余应力也随之消除, 消除的 载荷应为

$$
\begin{aligned}
& \left\{\begin{array}{l}
\boldsymbol{f}_{M X}=\int_{M} \sigma_{X}(z) \mathrm{d} z, \\
\boldsymbol{m}_{M X}=\int_{M} \sigma_{X}(z) z \mathrm{~d} z,
\end{array}\right. \\
& \left\{\begin{array}{l}
\boldsymbol{f}_{M Y}=\int_{M} \sigma_{Y}(z) \mathrm{d} z, \\
\boldsymbol{m}_{M Y}=\int_{M} \sigma_{Y}(z) z \mathrm{~d} z,
\end{array}\right.
\end{aligned}
$$

式中, $\boldsymbol{f}_{M X} 、 \boldsymbol{m}_{M X}$ 和 $\boldsymbol{f}_{M Y} 、 \boldsymbol{m}_{M Y}$ 分别为材料 $M$ 切除后毛坏 在轧制方向、横向方向上释放的力和力矩.

\section{2 加工变形}

由图2可知，材料去除前，无论是去除的材料 $M$ 还 是成形零件 $W$, 其受力均处于平衡状态，如式(1)和(2) 所示. 当毛坏释放 $\boldsymbol{f}_{M X} 、 \boldsymbol{m}_{M X}$ 和 $\boldsymbol{f}_{M Y} 、 \boldsymbol{m}_{M Y}$ 后, 成形零件 $W$ 的平衡状态被打破，必将受到外载的作用，其值可表 示为

$$
\left\{\begin{array}{l}
\boldsymbol{f}_{W X}=\int_{W} \sigma_{X}(z) \mathrm{d} z, \\
\boldsymbol{m}_{W X}=\int_{W} \sigma_{X}(z) z \mathrm{~d} z,
\end{array}\right.
$$




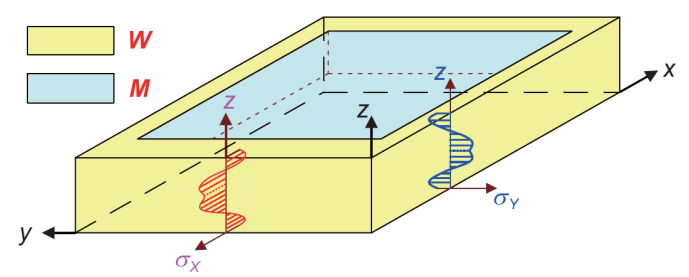

图 1 (网络版彩图)毛坏与零件示意图

Figure 1 (Color online) Diagram of a blank and its workpiece.

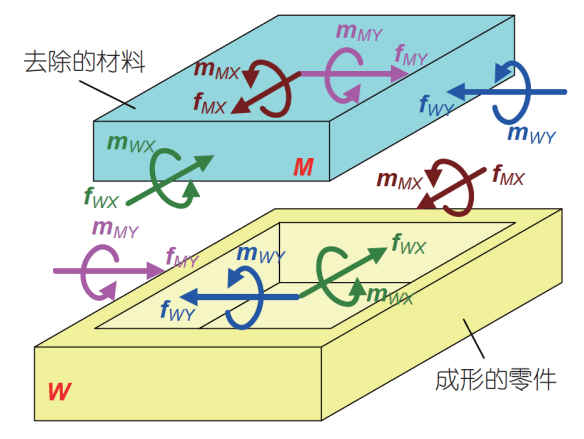

图 2 (网络版彩图)受力分析

Figure 2 (Color online) Force analysis of the workpiece.

$$
\left\{\begin{array}{l}
\boldsymbol{f}_{W Y}=\int_{W} \sigma_{Y}(z) \mathrm{d} z, \\
\boldsymbol{m}_{W Y}=\int_{W} \sigma_{Y}(z) z \mathrm{~d} z,
\end{array}\right.
$$

式中, $\boldsymbol{f}_{W X} 、 \boldsymbol{m}_{W X}$ 和 $\boldsymbol{f}_{W Y} 、 \boldsymbol{m}_{W Y}$ 分别为材料 $M$ 切除后, 零件 $W$ 在坐标系 $X Y Z$ 中受到的轧制方向、横向方向上的力 和力矩.

显然, 零件在受到 $\boldsymbol{f}_{W X} 、 \boldsymbol{m}_{W X}$ 和 $\boldsymbol{f}_{W Y} 、 \boldsymbol{m}_{W Y}$ 作用下, 势 必造成零件发生变形. 由式(5)和(6)可知，零件受到的 外载, 也是由零件内部残余应力 $\sigma_{Y}(z)$ 和 $\sigma_{X}(z)$ 引起的. 由 弹性理论可知, 在厚度方向上的任意位置 $z$ 处, 零件在 轧制方向的应变 $\varepsilon_{X}(z)$ 应为

$$
\varepsilon_{X}(z)=\frac{1}{E}\left[\sigma_{X}(z)-v \sigma_{Y}(z)\right]
$$

式中, $v$ 和 $E$ 分别为零件材料的泊松比和杨氏模量.

为了便于清晰地分析轧制方向的零件变形, 定义 轧制方向上的当量应力 $\bar{\sigma}_{X}(z)$ 如下:

$\bar{\sigma}_{X}(z)=\sigma_{X}(z)-v \sigma_{Y}(z)$.

因此, 当量应力 $\bar{\sigma}_{X}(z)$ 必将形成弯矩. 假定零件沿 轧制方向 $X$ 上位置 $x$ 处的中性层高度为 $z_{0}(x)$, 如图3 所 示. 若在中性层高度处建立中性层坐标系 $x y z$, 那么在

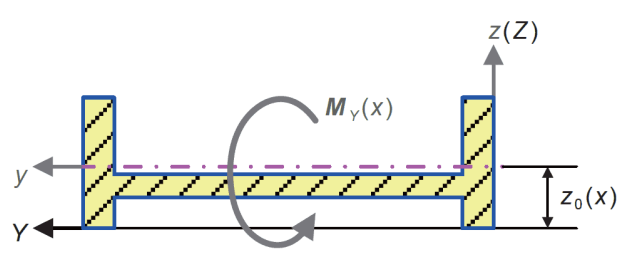

图 3 (网络版彩图)截面上的力矩计算参数确定

Figure 3 (Color online) Bend deformations of the workpiece.

坐标系 $x y z$ 中, 零件受到弯矩应为

$\boldsymbol{M}_{Y}(x)=\int_{W} \bar{\sigma}_{X}(x)\left[z-z_{0}(x)\right] \mathrm{d} z$.

弯矩 $\boldsymbol{M}_{Y}(x)$ 的正负符号规定如下: 使微段弯曲呈 凹形的弯矩为正. 这样, 由任意位置 $x$ 处截面上的弯矩 $\boldsymbol{M}_{Y}(x)$ 引起的厚向挠度 $\omega_{Z}(x)$ 可描述为

$\frac{d^{2} \omega_{Z}(x)}{d x^{2}}=\frac{M_{Y}(x)}{E I_{Y}(x)}$

式中, $I_{Y}(x)$ 为零件的截面惯性矩.

对式(10)进行二次积分, 可得任意位置 $x$ 处的挠 度为

$\omega_{Z}(x)=\int_{W} \frac{\boldsymbol{M}_{Y}(x)}{E I_{Y}(x)} d x^{2}+B x+C$.

将零件沿厚度方向 $Z$ 均匀地离散成 $n$ 层, 每层的厚 度为 $\Delta z$, 则式(9)中的力矩 $\boldsymbol{M}_{Y}(x)$ 可进一步描述为

$\boldsymbol{M}_{Y}(x)=\sum_{i=1}^{n} y_{i} \bar{\sigma}_{X}\left(z_{i}\right)\left[z_{0}(x)-z_{i}\right] \Delta z$,

式中, $\sigma_{q}^{\prime}=-\sigma_{h}^{\prime}, \sigma_{\text {max }}^{\prime}=-\sigma_{\text {min }}^{\prime}$ 分别为截面上第 $i(1 \leq i \leq n)$ 层有材料部位的宽度总和与中间高度值; $\bar{\sigma}_{X}\left(z_{i}\right)$ 为截面 上第 $i$ 层的当量应力值.

将式(12)代入式(11)后, 经整理可得任意位置 $x$ 处 的挠度为

$\omega_{Z}(x)=\sum_{i=1}^{n} \bar{\sigma}_{X}\left(z_{i}\right) \int_{W} \frac{y_{i}\left[z_{0}(x)-z_{i}\right] \Delta z}{E I_{Y}(x)} d x^{2}+B x+C$.

为了更清晰明了地理解式(13), 因此定义

$A_{i}(x)=\int_{W} \frac{y_{i}\left[z_{0}(x)-z_{i}\right] \Delta z}{E I_{Y}(x)} d x^{2}$.

显然, 当材料和工件结构确定后, $A_{i}(x)$ 为定值. 因 此, 变形量可进一步描述为 
$\omega_{Z}(x)=\sum_{i=1}^{n} A_{i}(x) \bar{\sigma}_{X}\left(z_{i}\right)+B x+C$.

飞机结构件大多为一维的梁类零件, 其变形的特 征为: 轧制方向的变形远大于其他方向的变形，且扭 转变形极小. 这样, 工件在 $A 、 B$ 两点处的弯曲变形被 约束(见图4), 故有

$$
\left\{\begin{array}{l}
\omega_{Z}\left(x_{A}\right)=\sum_{i=1}^{n} A_{i}\left(x_{A}\right) \bar{\sigma}_{X}\left(z_{i}\right)+B x_{A}+C=0, \\
\omega_{Z}\left(x_{B}\right)=\sum_{i=1}^{n} A_{i}\left(x_{B}\right) \bar{\sigma}_{X}\left(z_{i}\right)+B x_{B}+C=0 .
\end{array}\right.
$$

依据式(16), 利用消元法可得系数 $B$ 和 $C$ 为

$$
\left\{\begin{array}{l}
B=\frac{1}{\left(x_{A}-x_{B}\right)} \sum_{i=1}^{n}\left[A_{i}\left(x_{B}\right)-A_{i}\left(x_{A}\right)\right] \bar{\sigma}_{X}\left(z_{i}\right), \\
C=\frac{1}{\left(x_{A}-x_{B}\right)} \sum_{i=1}^{n}\left[x_{B} A_{i}\left(x_{A}\right)-x_{A} A_{i}\left(x_{B}\right)\right] \bar{\sigma}_{X}\left(z_{i}\right) .
\end{array}\right.
$$

代入式(15)后, 经整理可得任意位置 $x$ 处工件的挠 度为

$$
\begin{aligned}
\omega_{Z}(x)= & \sum_{i=1}^{n} A_{i}(x) \bar{\sigma}_{X}\left(z_{i}\right) \\
& +\frac{x}{\left(x_{A}-x_{B}\right)} \sum_{i=1}^{n}\left[A_{i}\left(x_{B}\right)-A_{i}\left(x_{A}\right)\right] \bar{\sigma}_{X}\left(z_{i}\right) \\
& +\frac{1}{\left(x_{A}-x_{B}\right)} \sum_{i=1}^{n}\left[x_{B} A_{i}\left(x_{A}\right)-x_{A} A_{i}\left(x_{B}\right)\right] \bar{\sigma}_{X}\left(z_{i}\right) .
\end{aligned}
$$

\section{3 残余应力规范}

实际加工中, 由于高速切削比常规切削的切削力 减小约 $30 \%{ }^{[12]}$, 产生的加工应力只存在于很薄的一层 内, 且时间短暂, 对初始残余应力的影响非常小, 从而 对最终的加工变形影响也非常小. 毛坏的初始残余应 力对零件变形起着决定性作用, 其贡献率高达 $90 \%$ 以 上 $^{[13]}$. 由此可见, 零件变形完全可以忽略加工过程中 产生的附加应力而仅受毛坏初始残余应力的影响 ${ }^{[14]}$. 若初始残余应力分布能够有效降低加工变形量，使得 零件变形量减小至规定范围内, 此时的初始残余应力 分布才被认为是合理的.

\section{1 变形因子}

为了更有规律地表达式(18)，这里引进一个新的

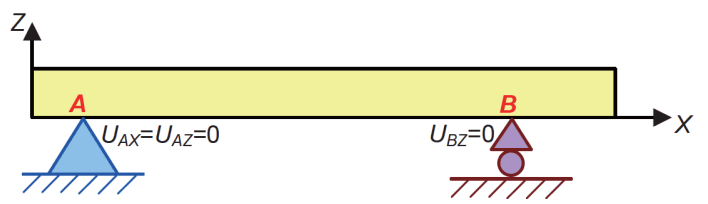

图 4 (网络版彩图)工件的弯曲变形

Figure 4 (Color online) Bend deformations of the workpiece.

概念, 即变形因子. 变形因子具体描述如下:

$$
\begin{aligned}
D_{Z i}(x)= & A_{i}(x)+\frac{A_{i}\left(x_{B}\right)-A_{i}\left(x_{A}\right)}{x_{A}-x_{B}} x \\
& +\frac{x_{B} A_{i}\left(x_{A}\right)-x_{A} A_{i}\left(x_{B}\right)}{x_{A}-x_{B}} .
\end{aligned}
$$

将式(19)代入式(18), 则弯曲变形量的计算式可简 化为

$\omega_{Z}(x)=\sum_{i=u}^{v} D_{Z i}(x) \bar{\sigma}_{X}\left(z_{i}\right)$.

由式(20)的变形模型可知，若第 $k$ 层的残余应力为 1 , 其余各层的残余应力均为 0 , 则此时计算得到的弯 曲变形(称之为单位变形), 其大小即为第 $k$ 层的变形因 子 $D_{Z k}(x)$, 即

$$
\omega_{Z k}(x)=D_{Z k}(x) \times 1+\sum_{\substack{i=u \\ i \neq k}}^{v} D_{Z i}(x) \times 0=D_{Z k}(x) .
$$

一般采用裂纹柔度法、钻孔法等方法, 能够测出 毛坏(即铝厚板)内的初始残余应力分布状况. 实际加 工过程中，一般是先对毛坏上、下两面进行光面处理， 再粗加工各框等其余表面, 最后精加工各面. 因此, 沿 厚度方向找出零件在毛坏中的位置, 即可获得应 力 $\bar{\sigma}_{X k}(z)(1 \leq u \leq k \leq v \leq n)$, 如图5中应力曲线的阴影部分.

这样, 由式(21)可知, 若求出各层单位变形就可计 算出变形因子 $D_{Z}(x)$, 这里利用有限元方法计算单位变 形. 为了提高计算效率, 可在有限元软件ABAQUS主 窗口的命令行接口，按照图6所示的流程键入Python命 令和相应的数学计算表达式即可.

尽管飞机结构件大多为梁类等一维零件, 但事实 上还有肋板类等二维零件. 对于结构复杂的二维飞机 整体结构件, 在不同方向上抵抗变形的能力也不相同, 对应的变形因子也不同.

结合式(20)和(8), 依据胡克定律可知, 飞机结构件 的厚向变形可表示为 


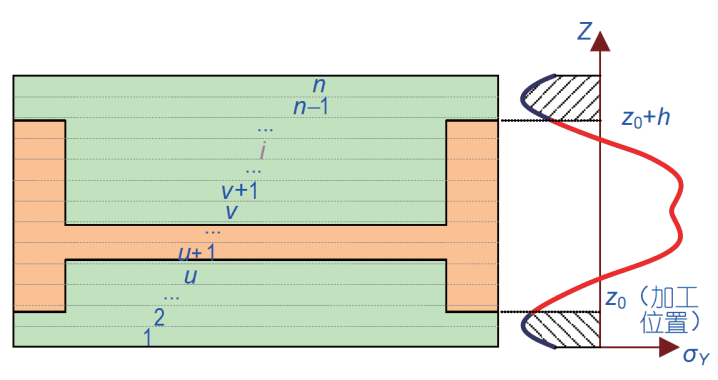

图 5 (网络版彩图)毛坏初始残余应力与零件残余应力的关系 Figure 5 (Color online) Relationship between residual stresses in blank and ones in workpiece.

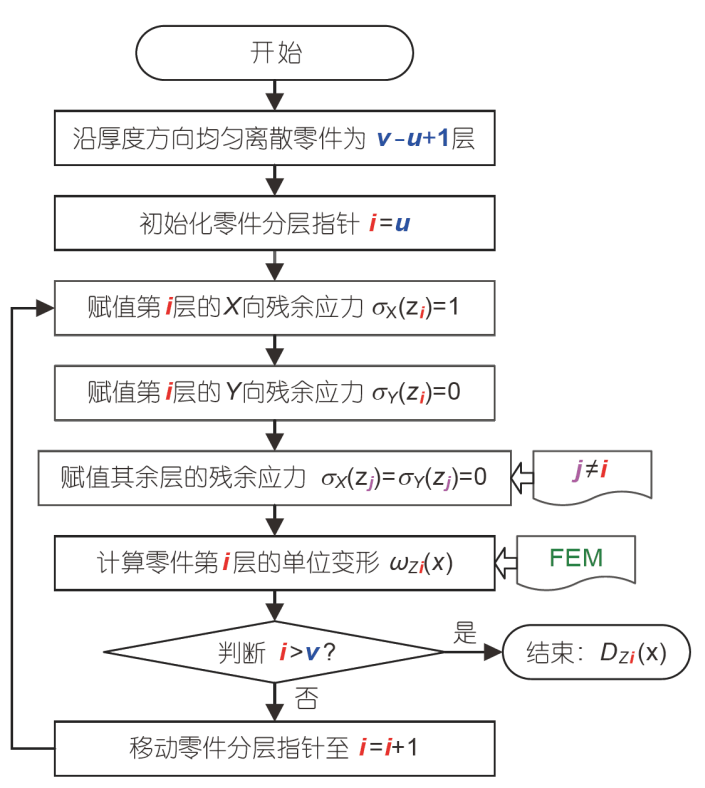

图 6 (网络版彩图)变形因子的计算流程

Figure 6 (Color online) Flowchart of calculating the deformation factor.

$$
\left\{\begin{array}{l}
\omega_{Z}(x)=\sum_{i=u}^{v} D_{Z i}(x) \bar{\sigma}_{X}\left(z_{i}\right), \\
\omega_{Z}(y)=\sum_{i=u}^{v} D_{Z i}(y) \bar{\sigma}_{Y}\left(z_{i}\right),
\end{array}\right.
$$

式中, $D_{Z i}(x), D_{Z i}(y)(u \leq i \leq v)$ 分别为轧制方向、横向方 向上的变形因子; $\bar{\sigma}_{X}\left(z_{i}\right), \bar{\sigma}_{Y}\left(z_{i}\right)$ 分别为当量应力.

基于飞机整体结构件属于小变形问题这一事实, 认为飞机结构件在任意点 $(x, y)$ 处的变形是轧制和横向 两个方向变形影响的叠加, 即有

$$
\omega_{Z}(x, y)=\sum_{i=u}^{v} D_{Z i}(x) \bar{\sigma}_{X}\left(z_{i}\right)+\sum_{i=u}^{v} D_{Z i}(y) \bar{\sigma}_{Y}\left(z_{i}\right) .
$$

类似于式(20), 只要计算出式(23)中的两个变形因
子 $D_{Z i}(x)$ 和 $D_{Z i}(y)$, 就可求出弯曲变形 $v_{Z}^{2}$.

\section{2 应力规范模型}

由式(20)和(23)可知, 通过单位力方法容易求得变 形因子. 此时应以实际的加工要求 $2 \Delta_{Z}$ 为界限, 初始残 余应力引起的零件变形, 必须满足下列条件, 即

$$
-\Delta_{Z} \leq \sum_{i=u}^{v} D_{Z i}(x) \bar{\sigma}_{X}\left(z_{i}\right) \leq \Delta_{Z}
$$

或

$$
-\Delta_{Z} \leq \sum_{i=u}^{v} D_{Z i}(x) \bar{\sigma}_{X}\left(z_{i}\right)+\sum_{i=u}^{v} D_{Z i}(y) \bar{\sigma}_{Y}\left(z_{i}\right) \leq \Delta_{Z} .
$$

由于毛坏初始残余应力具有自平衡特性, 因此, 控 制应力函数也应具有自平衡特性, 故存在下列关系:

$$
\begin{aligned}
& \left\{\begin{array}{l}
\sum_{i=1}^{n} \bar{\sigma}_{X}\left(z_{i}\right)=0, \\
\sum_{i=1}^{n} \bar{\sigma}_{X}\left(z_{i}\right) z_{i}=0,
\end{array}\right. \\
& \left\{\begin{array}{l}
\sum_{i=1}^{n} \bar{\sigma}_{Y}\left(z_{i}\right)=0, \\
\sum_{i=1}^{n} \bar{\sigma}_{Y}\left(z_{i}\right) z_{i}=0 .
\end{array}\right.
\end{aligned}
$$

由夹逼原理可知, 式(26)和(27)可等效地表示为下 列不等式:

$$
\begin{aligned}
& \left\{\begin{array}{l}
0 \leq \sum_{i=1}^{n} \bar{\sigma}_{X}\left(z_{i}\right) \leq 0, \\
0 \leq \sum_{i=1}^{n} \bar{\sigma}_{X}\left(z_{i}\right) z_{i} \leq 0,
\end{array}\right. \\
& \left\{\begin{array}{l}
0 \leq \sum_{i=1}^{n} \bar{\sigma}_{Y}\left(z_{i}\right) \leq 0, \\
0 \leq \sum_{i=1}^{n} \bar{\sigma}_{Y}\left(z_{i}\right) z_{i} \leq 0 .
\end{array}\right.
\end{aligned}
$$

根据实际的加工要求, 确定出零件上待控制变形 的 $m$ 个位置, 即 $x=x_{1}, x_{2}, \ldots, x_{m}$ 和 $y=y_{1}, y_{2}, \ldots, y_{m}$, 联合式 (24), (25)与式(28), (29), 分别获得如下关于应力的齐 次线性不等式方程组.

$A x \leq 0$,

式中, 若记 $\sigma_{n+1}=1$, 当零件属于梁类等一维结构时, 残 余应力 $\boldsymbol{x}=\left[\sigma_{1}, \ldots, \sigma_{u-1}, \sigma_{u}, \ldots, \sigma_{v}, \sigma_{v+1}, \ldots, \sigma_{n}, \sigma_{n+1}\right]^{\mathrm{T}}$, $\sigma_{i}=\bar{\sigma}_{X}\left(z_{i}\right)$, 系数矩阵为 
$\boldsymbol{A}=\left[\begin{array}{cccccccccc}D_{11} & \cdots & D_{u-1,1} & D_{u, 1} & \cdots & D_{v, 1} & D_{v+1,1} & \cdots & D_{n, 1} & -\Delta_{Z} \\ D_{12} & \cdots & D_{u-1,2} & D_{u, 2} & \cdots & D_{v, 2} & D_{v+1,2} & \cdots & D_{n, 2} & -\Delta_{Z} \\ \vdots & \cdots & \vdots & \vdots & \cdots & \vdots & \vdots & \cdots & \vdots & \vdots \\ D_{1, m} & \cdots & D_{u-1, m} & D_{u, m} & \cdots & D_{v, m} & D_{v+1, m} & \cdots & D_{n, m} & -\Delta_{Z} \\ -D_{11} & \cdots & -D_{u-1,1} & -D_{u, 1} & \cdots & -D_{v, 1} & -D_{v+1,1} & \cdots & -D_{n, 1} & -\Delta_{Z} \\ -D_{12} & \cdots & -D_{u-1,2} & -D_{u, 2} & \cdots & -D_{v, 2} & -D_{v+1,2} & \cdots & -D_{n, 2} & -\Delta_{Z} \\ \vdots & \cdots & \vdots & \vdots & \cdots & \vdots & \vdots & \cdots & \vdots & \vdots \\ -D_{1, m} & \cdots & -D_{u-1, m} & -D_{u, m} & \cdots & -D_{v, m} & -D_{v+1, m} & \cdots & -D_{n, m} & -\Delta_{Z} \\ z_{1} & \cdots & z_{u-1} & z_{u} & \cdots & z_{v} & z_{v+1} & \cdots & z_{n} & 0 \\ -z_{1} & \cdots & -z_{u-1} & -z_{u} & \cdots & -z_{v} & -z_{v+1} & \cdots & -z_{n} & 0 \\ 1 & \cdots & 1 & 1 & \cdots & 1 & 1 & \cdots & 1 & 0 \\ -1 & \cdots & -1 & -1 & \cdots & -1 & -1 & \cdots & -1 & 0\end{array}\right]$

$1 \leq j \leq m \cdot D_{i, j}=D_{Z i}\left(x_{j}\right), 1 \leq i \leq n$.

而当零件属于板类等二维结构时, 残余应力 $\boldsymbol{x}=\left[\sigma_{1}, \ldots, \sigma_{u-1}, \sigma_{u}, \ldots, \sigma_{v}, \sigma_{v+1}, \ldots, \sigma_{n}, \sigma_{1}^{\prime}, \ldots, \sigma_{u-1}^{\prime}, \sigma_{u}^{\prime}, \ldots, \sigma_{v}^{\prime}\right.$, $\left.\sigma_{v+1}^{\prime}, \ldots, \sigma_{n}^{\prime}, \sigma_{n+1}\right]^{\mathrm{T}}, \sigma_{i}=\bar{\sigma}_{X}\left(z_{i}\right), \sigma_{i}^{\prime}=\bar{\sigma}_{Y}\left(z_{i}\right)$, 系数矩阵则为

$\boldsymbol{A}=\left[\begin{array}{ccccccccccccccc}D_{11} & \cdots & D_{u, 1} & \cdots & D_{v, 1} & \cdots & D_{n, 1} & D_{11}^{\prime} & \cdots & D_{u, 1}^{\prime} & \cdots & D_{v, 1}^{\prime} & \cdots & D_{n, 1}^{\prime} & -\Delta_{Z} \\ D_{12} & \cdots & D_{u, 2} & \cdots & D_{v, 2} & \cdots & D_{n, 2} & D_{12}^{\prime} & \cdots & D_{u, 2}^{\prime} & \cdots & D_{v, 2}^{\prime} & \cdots & D_{n, 2}^{\prime} & -\Delta_{Z} \\ \vdots & \cdots & \vdots & \cdots & \vdots & \cdots & \vdots & \vdots & \cdots & \vdots & \cdots & \vdots & \cdots & \vdots & \vdots \\ D_{1, m} & \cdots & D_{u, m} & \cdots & D_{v, m} & \cdots & D_{n, m} & D_{1, m}^{\prime} & \cdots & D_{u, m}^{\prime} & \cdots & D_{v, m}^{\prime} & \cdots & D_{n, m}^{\prime} & -A_{Z} \\ -D_{11} & \cdots & -D_{u, 1} & \cdots & -D_{v, 1} & \cdots & -D_{n, 1} & -D_{11}^{\prime} & \cdots & -D^{\prime}{ }_{u, 1} & \cdots & -D^{\prime}{ }_{v, 1} & \cdots & -D_{n, 1}^{\prime} & -\Delta_{Z} \\ -D_{12} & \cdots & -D_{u, 2} & \cdots & -D_{v, 2} & \cdots & -D_{n, 2} & -D_{12}^{\prime} & \cdots & -D^{\prime}{ }_{u, 2} & \cdots & -D_{v, 2}^{\prime} & \cdots & -D_{n, 2}^{\prime} & -\Delta_{Z} \\ \vdots & \cdots & \vdots & \cdots & \vdots & \cdots & \vdots & \vdots & \cdots & \vdots & \cdots & \vdots & \cdots & \vdots & \vdots \\ -D_{1, m} & \cdots & -D_{u, m} & \cdots & -D_{v, m} & \cdots & -D_{n, m} & -D_{1, m}^{\prime} & \cdots & -D^{\prime}{ }_{u, m} & \cdots & -D_{v, m}^{\prime} & \cdots & -D^{\prime}{ }_{n, m} & -\Delta_{Z} \\ z_{1} & \cdots & z_{u} & \cdots & z_{v} & \cdots & z_{n} & 0 & \cdots & 0 & \cdots & 0 & \cdots & 0 & 0 \\ -z_{1} & \cdots & -z_{u} & \cdots & -z_{v} & \cdots & -z_{n} & 0 & \cdots & 0 & \cdots & 0 & \cdots & 0 & 0 \\ 0 & \cdots & 0 & \cdots & 0 & \cdots & 0 & z_{1}^{\prime} & \cdots & z_{u}^{\prime} & \cdots & z_{v}^{\prime} & \cdots & z_{n}^{\prime} & 0 \\ 0 & \cdots & 0 & \cdots & 0 & \cdots & 0 & -z_{1}^{\prime} & \cdots & -z_{u}^{\prime} & \cdots & -z^{\prime} & \cdots & -z_{n}^{\prime} & 0 \\ 1 & \cdots & 1 & \cdots & 1 & \cdots & 1 & 0 & \cdots & 0 & \cdots & 0 & \cdots & 0 & 0 \\ -1 & \cdots & -1 & \cdots & -1 & \cdots & -1 & 0 & \cdots & 0 & \cdots & 0 & \cdots & 0 & 0 \\ 0 & \cdots & 0 & \cdots & 0 & \cdots & 0 & 1 & \cdots & 1 & \cdots & 1 & \cdots & 1 & 0 \\ 0 & \cdots & 0 & \cdots & 0 & \cdots & 0 & -1 & \cdots & -1 & \cdots & -1 & \cdots & -1 & 0\end{array}\right]$,

$D_{i, j}=D_{Z i}\left(x_{j}\right), D_{i, j}^{\prime}=D_{Z i}\left(y_{j}\right)$.

\section{3 枢轴变换方法}

式(30)中的变量 $\boldsymbol{x}$ 即为应力, 采用枢轴变换方法可 进行求解. 不等式(30)的解的一般形式 ${ }^{[15]}$ 通常表示为

$$
\boldsymbol{x}=\boldsymbol{V} \boldsymbol{\zeta}+\boldsymbol{W \rho},
$$

其中, $\zeta$ 为任意的系数向量, $\boldsymbol{\rho}$ 为任意的非负系数向量, $V, W$ 分别为基向量矩阵.
显然, 为了获得 $\boldsymbol{x}$ 的通解, 关键在于解算出基向量 矩阵 $\boldsymbol{V}$ 和 $\boldsymbol{W}$.

若记系数矩阵 $\boldsymbol{A}=\left(a_{i j}\right)_{M \times N}$ 的行数、列数分别为 $M$, $N($ 一维结构有 $M=2 m+4, N=n+1$; 而二维结构有 $M=2 m$ $+8, N=2 n+1)$, 则由 $\boldsymbol{A}$ 既可生成凸锥 $\boldsymbol{A}_{\rho}=\left\{\boldsymbol{x} \mid \boldsymbol{x}=\rho_{1} \boldsymbol{a}_{1}+\rho_{2} \boldsymbol{a}_{2}\right.$ $\left.+\ldots+\rho_{M} \boldsymbol{a}_{M}\right\}$, 也可生成线性空间 $\boldsymbol{A}_{\zeta}=\left\{\boldsymbol{y} \mid \boldsymbol{y}=\zeta_{1} \boldsymbol{a}_{1}+\zeta_{2} \boldsymbol{a}_{2}+\ldots\right.$ $\left.+\zeta_{M} \boldsymbol{a}_{M}\right\} \quad\left(\rho_{i} \geq 0,1 \leq i \leq M\right)$. 再记矩阵 $\boldsymbol{B}=\left\{\boldsymbol{a}_{i} \mid \boldsymbol{a}_{i} \in \boldsymbol{A}_{\rho}\right.$, 且 $-\boldsymbol{a}_{i}$ 
$\left.\in \boldsymbol{A}_{\rho}\right\}, \boldsymbol{C}=\left\{\boldsymbol{a}_{i} \mid \boldsymbol{a}_{i} \in \boldsymbol{A}_{\rho}\right.$, 但 $\left.-\boldsymbol{a}_{i} \notin \boldsymbol{A}_{\rho}\right\}$, 那么求解基向量矩阵 $\boldsymbol{V}$ 和 $\boldsymbol{W}$ 的旋转算法如图7所示.

值得注意的是, 按照枢轴变换算法求出基向量矩 阵 $\boldsymbol{V}$ 和 $\boldsymbol{W}$ 后, 将 $x_{N}=1$ 代入式(30), 经整理可得合理的毛 坏初始残余应力.

\section{4 规范实例}

薄板件的材料选用经过拉伸 (拉伸率为 $\delta=2.4 \%$ ) 的 7075-T73铝合金板材 ${ }^{[16]}$, 其弹性模量 $E=70.5 \mathrm{GPa}$, 泊松 比 $v=0.33$. 毛坏尺寸长 $L=200 \mathrm{~mm}$, 宽 $W=20 \mathrm{~mm}$, 高 $H=6 \mathrm{~mm}$, 而铣削成形的薄板件尺寸长 $l^{\prime}=200 \mathrm{~mm}$, 宽 $w^{\prime}=20 \mathrm{~mm}$, 高 $h^{\prime}=4.5 \mathrm{~mm}$, 要求变形不超过 $0.1 \mathrm{~mm}$, 如 图8(a)所示.

\section{步骤一, 残余应力测量}

由于横向方向 $Y$ 的残余应力很小, 可忽略不计, 毛 坯内轧制方向 $X$ 的初始残余应力分布曲线如图 8(b) 所示.

沿厚度方向 $Z$ 对薄板件进行分层 $n=20$, 每层厚度 均为 $\Delta z=0.3 \mathrm{~mm}$. 若选取每层中心的残余应力作为该 层的应力值, 则根据图 $8(\mathrm{~b})$ 的应力分布状态, 通过静力 平衡条件求解出每层的应力值如表 1 所示.

\section{步骤二, 加工变形计算}

显然, 由于薄板件沿轧制方向 $X$ 的任意位置 $x$ 处截
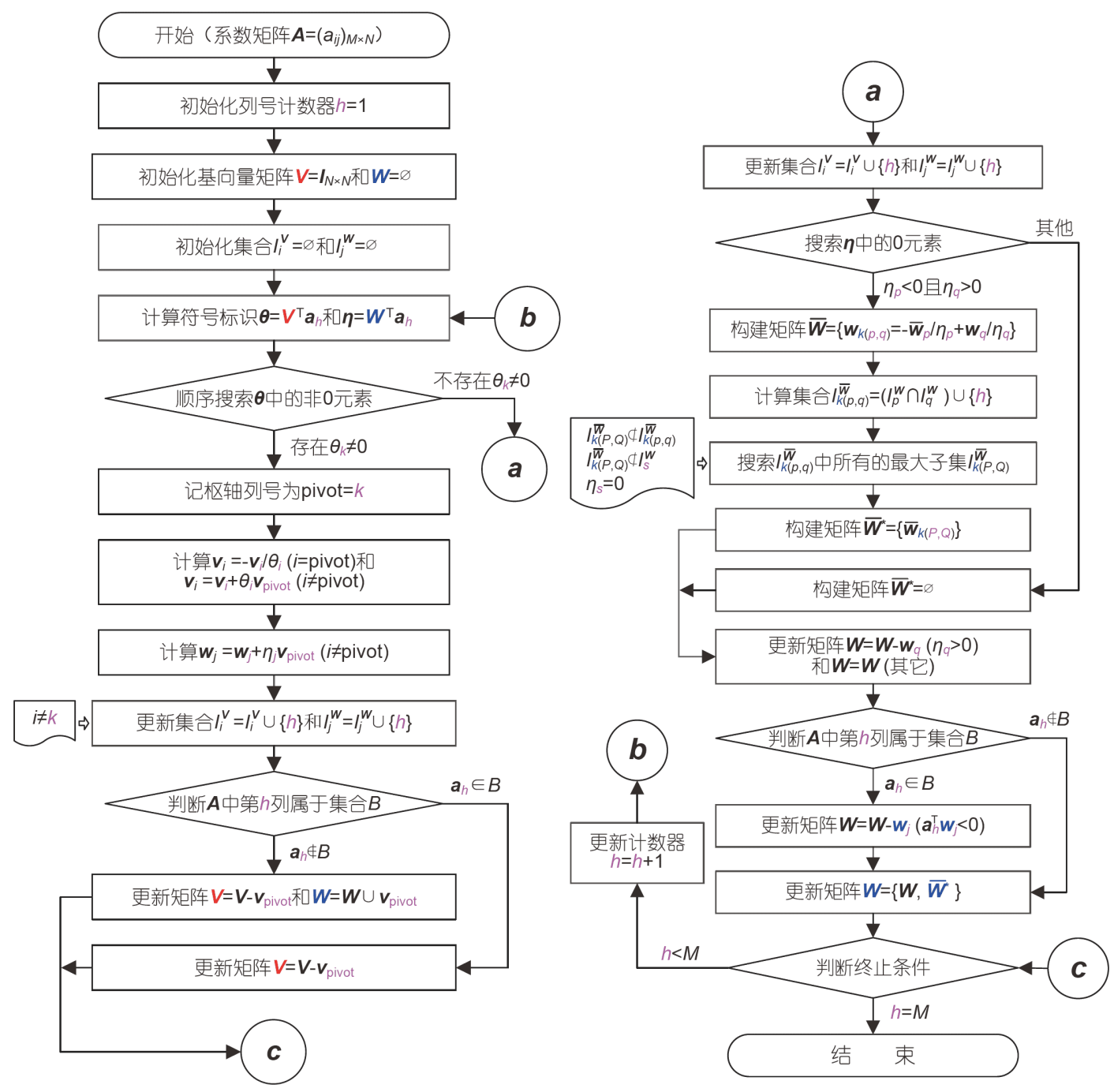

图 7 (网络版彩图)基向量的求解流程

Figure 7 (Color online) Solution flowchart of basis vectors. 

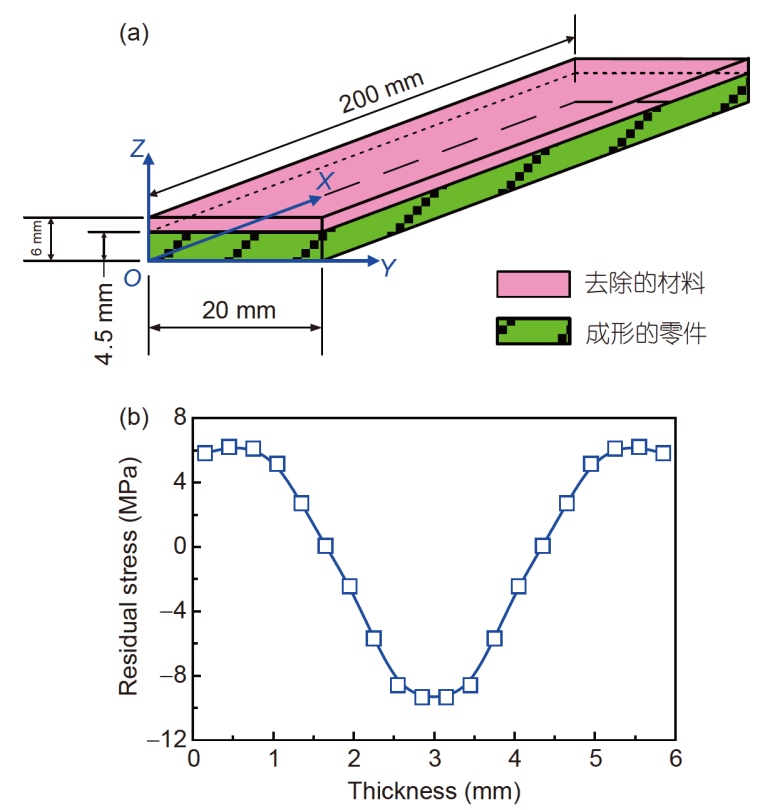

图 8 (网络版彩图)薄板件毛坏初始残余应力. (a) 薄板件; (b) 初始残余应力

Figure 8 (Color online) Initial residual stress in blank of thin-walled plate. (a) Thin-walled plate; (b) initial residual stresses.

面均相同, 故中性轴和截面惯性矩分别为 $z_{0}(x)=$ $2.25 \mathrm{~mm}$ 和 $I_{Y}(x)=151.875 \mathrm{~mm}^{4}$, 则由式(15)可知截面力 矩均为

$\boldsymbol{M}_{Y}(x)=\sum_{i=1}^{15} w^{\prime} z_{i} \bar{\sigma}_{X}\left(z_{i}\right)\left(z_{i} i-\frac{z_{i}}{2}-z_{0}(x)\right) \approx-480.7$.

因此，根据式(18)可得关于轧制方向的挠曲方程
如下:

$\omega_{Z}(x)=-2.245 \times 10^{-5} x^{2}+4.49 \times 10^{-3} x$.

采用有限元方法分析时, 单元类型选用CPS8R, 网 格密度设定为 0.3 ，总共有 53320 个单元. 选择“Static, General”分析步类型后，使用Model change进行“单元 杀死”模拟材料的去除，计算后得到的变形云图如图9 所示.

\section{步骤三, 变形实验验证}

选用Fadal VMC 3016L立式铣床进行加工，刀具 直径 $\Phi=50 \mathrm{~mm}$, 转速 $n=5000 \mathrm{r} / \mathrm{min}$, 如图 10 所示, 这些 参数可保证在加工过程中引入的残余应力水平很小.

变形测量直接在同一台铣床上进行，测量头可直 接安装在刀架上 ${ }^{[16]}$, 如图11所示. 为了避免测量的随 机性，反复测量加工区域内同一位置处的工件变形, 使测量精度能够满足误差在 $0.01 \mathrm{~mm}$ 之内的实验需求. 为了使加工测试过程与航空业界零件制造的现场工作

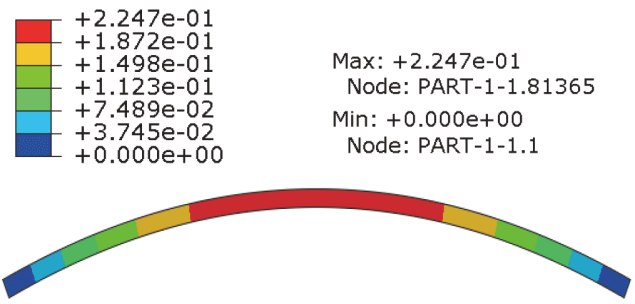

图 9 (网络版彩图)仿真变形结果

Figure 9 (Color online) Simulated results of deformations.

表 1 薄板件毛坏的残余应力值

Table 1 Residual stresses in blank of thin-walled plate

\begin{tabular}{ccc||ccc}
\hline 层号 $i$ & 位置 $z_{i}(\mathrm{~mm})$ & 等效残余应力 $\bar{\sigma}_{X}\left(z_{i}\right)(\mathrm{MPa})$ & 层号 $i$ & 位置 $z_{i}(\mathrm{~mm})$ & 等效残余应力 $\bar{\sigma}_{X}\left(z_{i}\right)(\mathrm{MPa})$ \\
\hline 1 & 0.15 & 5.81 & 11 & 3.15 & -9.34 \\
2 & 0.45 & 6.21 & 12 & 3.45 & -8.59 \\
3 & 0.75 & 6.11 & 13 & 3.75 & -5.69 \\
4 & 1.05 & 5.16 & 14 & 4.05 & 0.06 \\
5 & 1.35 & 2.71 & 15 & 4.35 & 2.71 \\
6 & 1.65 & 0.06 & 16 & 4.65 & 5.16 \\
7 & 1.95 & -2.44 & 17 & 4.95 & 6.11 \\
8 & 2.25 & -5.69 & 18 & 5.25 & 6.21 \\
9 & 2.55 & -8.59 & 19 & 5.55 & 5.81 \\
\hline
\end{tabular}




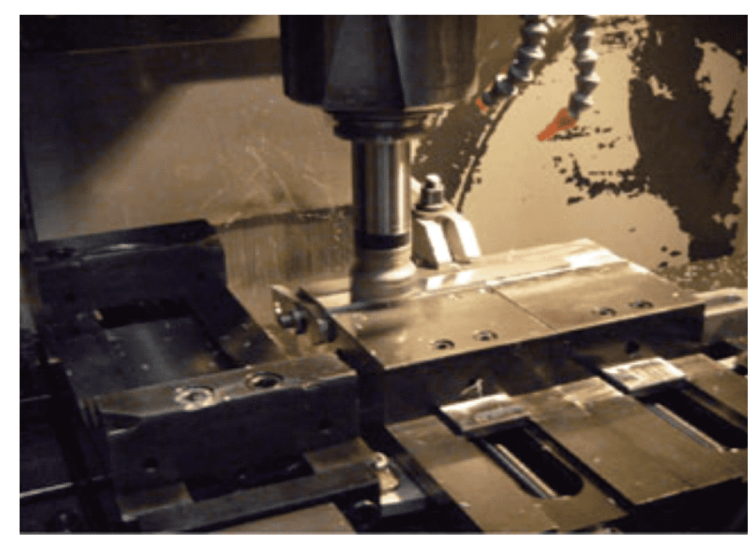

图 10 (网络版彩图)零件的实际加工

Figure 10 (Color online) Practical machining of the workpiece.

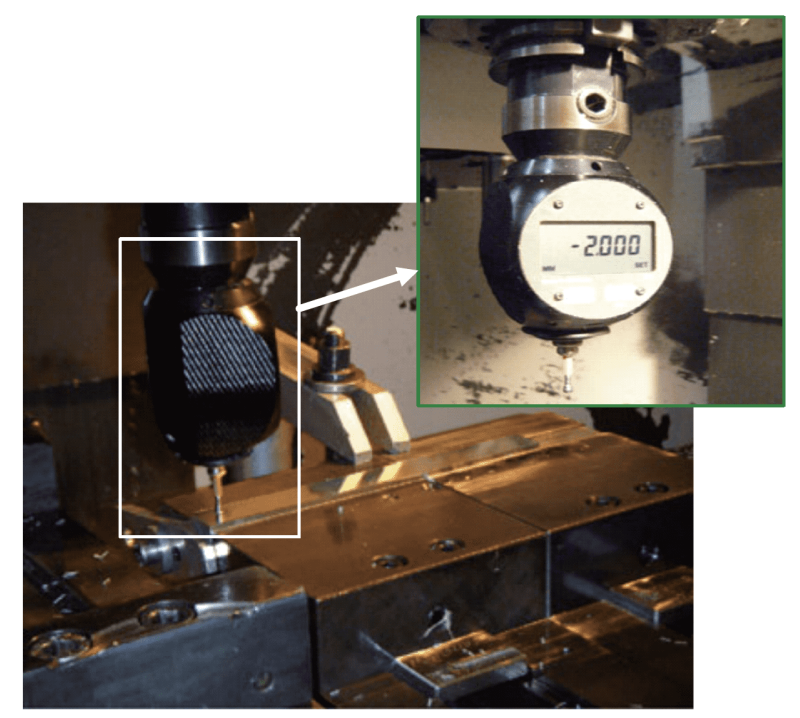

图 11 (网络版彩图)变形测量方案

Figure 11 (Color online) Measurement scheme of deformations.

流尽量吻合, 每次加工去除一层材料后, 测量加工变形 和工件厚度直到下一次加工准备完成，中间花费 $3 \sim 5 \mathrm{~min}$.

实验测量、有限元仿真和模型求解获得的挠度曲 线比较如图12所示, 三者趋势完全一致, 最大变形位置 均发生在薄板件沿长度方向的正中间位置，即在长度 方向的 $100 \mathrm{~mm}$ 处, 实验测量值为 $0.18 \mathrm{~mm}$, 有限元仿真 值为 $0.22 \mathrm{~mm}$, 而模型计算结果也为 $0.22 \mathrm{~mm}$. 模型计 算值和有限元仿真值高度统一，两者与实验结果的相 对误差为 $22.22 \%$.

引起误差的原因, 除了高速铣削在动态加工过程

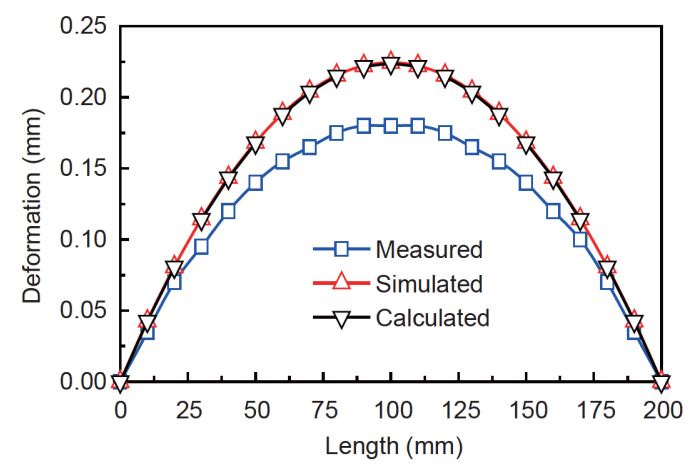

图 12 (网络版彩图)变形比较

Figure 12 (Color online) Comparison of deformations.

中引入了一定的残余应力, 主要原因如下:

(1) 残余应力和变形测量过程中, 人为操作、设备 条件和实验方法本身会不可避免地带来误差;

(2) 铝厚板加工变形分析过程中认为, 处于同一厚 度的不同位置, 残余应力是完全相同的. 但事实上同一 平面内存在或多或少的差异, 这也将使得计算结果产 生偏差;

(3) 有限元仿真计算中, 尤其是大型复杂零件, 网 格密度受硬件限制而无法太高, 亦将造成一定的计算 误差.

\section{步骤四, 应力规范制订}

由毛坏初始残余应力引起的最大变形为 $0.22 \mathrm{~mm}$, 超出了 $0.1 \mathrm{~mm}$ 的加工要求范围, 为此, 采取枢轴变换方 法调控毛坏的初始残余应力. 通过实验验证的有限元 方法和模型法, 均可有效地计算出变形因子. 因此, 根 据式(19)可得各层的变形因子为

$D_{Z i}(x)=\frac{3 \times\left(2.25-z_{i}\right)}{70.5 \times 151.875 \times 10^{3}}\left(x^{2}-200 x\right)$.

零件沿厚度方向 $Z$ 从 $z=0$ 至 $z=4.5 \mathrm{~mm}$ 共分为 15 层, 考虑距离端面分别为 $x=50 \mathrm{~mm}$ 和 $x=100 \mathrm{~mm}$ 位置的 $A, B$ 两点, 通过逐层施加单位力, 利用有限元方法计算的单 位变形(即变形因子)如表2所示.

由于薄板件的毛坏初始残余应力分布和结构均呈 对称形式, $A, B$ 两点的变形线性相关, 因此, 仅需控制 $A$ 点变形不超出 $\Delta z=50 \times 10^{-3} \mathrm{~mm}$ 即可. 结合表 2 的数据, 根据式(31) 可知应力规范模型中 $x_{21}=1$, 且系数矩阵 $\boldsymbol{A}$ 如表3所示.

根据表 3 的系数矩阵 $\boldsymbol{A}$, 可知 $\boldsymbol{B}=\left\{\boldsymbol{a}_{3}, \boldsymbol{a}_{4}, \boldsymbol{a}_{5}, \boldsymbol{a}_{6}\right\}, \boldsymbol{C}=$ $\left\{\boldsymbol{a}_{1}, \boldsymbol{a}_{2}\right\}$. 这样, 依据图7的流程, 采用枢轴变换方法计 
表 2 薄板件的变形因子

Table 2 Deformation factor of thin-walled plate

\begin{tabular}{|c|c|c|c|c|c|c|c|}
\hline \multirow{2}{*}{ 层号 $i$} & \multirow{2}{*}{ 位置 $z_{i}(\mathrm{~mm})$} & \multicolumn{2}{|c|}{$D_{Z i}(x)\left(\times 10^{-3} \mathrm{~mm}\right)$} & \multirow{2}{*}{ 层号 $i$} & \multirow{2}{*}{ 位置 $z_{i}(\mathrm{~mm})$} & \multicolumn{2}{|c|}{$D_{Z i}(x)\left(\times 10^{-3} \mathrm{~mm}\right)$} \\
\hline & & $x=100 \mathrm{~mm}$ & $x=50 \mathrm{~mm}$ & & & $x=100 \mathrm{~mm}$ & $x=50 \mathrm{~mm}$ \\
\hline 1 & 0.15 & 5.8839 & 4.4129 & 9 & 2.55 & -0.8406 & -0.6304 \\
\hline 2 & 0.45 & 5.0433 & 3.7825 & 10 & 2.85 & -1.6811 & -1.2608 \\
\hline 3 & 0.75 & 4.2028 & 3.1521 & 11 & 3.15 & -2.5217 & -1.8913 \\
\hline 4 & 1.05 & 3.3622 & 2.5217 & 12 & 3.45 & -3.3622 & -2.5217 \\
\hline 5 & 1.35 & 2.5217 & 1.8913 & 13 & 3.75 & -4.2028 & -3.1521 \\
\hline 6 & 1.65 & 1.6811 & 1.2608 & 14 & 4.05 & -5.0433 & -3.7825 \\
\hline 7 & 1.95 & 0.8406 & 0.6304 & 15 & 4.35 & -5.8839 & -4.4129 \\
\hline 8 & 2.25 & 0 & 0 & - & - & - & - \\
\hline
\end{tabular}

表 3 薄板件的系数矩阵 $\left(\times 10^{-3} \mathrm{~mm}\right)$

Table 3 Coefficient matrix of thin-walled plate $\left(\times 10^{-3} \mathrm{~mm}\right)$

\begin{tabular}{ccccccccccccc}
\hline 列号 & 1 & 2 & 3 & 4 & 5 & 6 & 7 & 8 & 9 & 10 & 11 \\
\hline 1 & 5.8839 & 5.0433 & 4.2028 & 3.3622 & 2.5217 & 1.6811 & 0.8406 & 0 & -0.8406 & -1.6811 & -2.5217 \\
2 & -5.8839 & -5.0433 & -4.2028 & -3.3622 & -2.5217 & -1.6811 & -0.8406 & 0 & 0.8406 & 1.6811 & 2.5217 \\
3 & 0.15 & 0.45 & 0.75 & 1.05 & 1.35 & 1.65 & 1.95 & 2.25 & 2.55 & 2.85 & 3.15 & -2.5 \\
4 & -0.15 & -0.45 & -0.75 & -1.05 & -1.35 & -1.65 & -1.95 & -2.25 & -2.55 & -2.85 & -3.15 & 1 \\
5 & 1 & 1 & 1 & 1 & 1 & 1 & 1 & 1 & 1 & 1 & -1 \\
6 & -1 & -1 & -1 & -1 & -1 & -1 & -1 & -1 & -1 & -1 \\
\hline \hline 列号 & 12 & 13 & 14 & 15 & 16 & 17 & 18 & 19 & 20 & 21 \\
1 & -3.3622 & -4.2028 & -5.0433 & -5.8839 & 0 & 0 & 0 & 0 & 0 & -50 \\
2 & 3.3622 & 4.2028 & 5.0433 & 5.8839 & 0 & 0 & 0 & 0 & 0 & -50 \\
3 & 3.45 & 3.75 & 4.05 & 4.35 & 4.65 & 4.95 & 5.25 & 5.55 & 5.85 & 0 \\
4 & -3.45 & -3.75 & -4.05 & -4.35 & -4.65 & -4.95 & -5.25 & -5.55 & -5.85 & 0 \\
5 & 1 & 1 & 1 & 1 & 1 & 1 & 1 & 1 & 1 & 0 \\
6 & -1 & -1 & -1 & -1 & -1 & -1 & -1 & -1 & -1 & 0 \\
\hline
\end{tabular}

算基向量的详细步骤如下.

首先, 始化基向量.

$h=1$.

$\boldsymbol{V}=\boldsymbol{I}_{21 \times 21}, \boldsymbol{W}=\Phi$.

$I_{i}^{\mathbf{V}}=\Phi(1 \leq i \leq 21)$.

其次, 确定旋转枢轴.

$\boldsymbol{\theta}=\boldsymbol{V}^{\mathrm{T}} \boldsymbol{a}_{1}=[5.8839,5.0433,4.2028,3.3622,2.5217$, $1.6811,0.8406,0,-0.8406,-1.6811,-2.5217,-3.3622$, $-4.2028,-5.0433,-5.8839,0,0,0,0,0,-50]^{\mathrm{T}}$.

顺序搜索 $\boldsymbol{\theta}$ 中的第一个非零元素 $\theta_{1}=5.8839$, 故 $\boldsymbol{V}$ 中
第一列(即 $k=1)$ 为枢轴列, 即 $\mathrm{pivot}=1$.

再次, 执行枢轴变换.

进行第一次旋转变换后, 添加 $h=1$ 至 $I_{i} \mathbf{v}$ 中, 即 $I_{i} \mathbf{v}=$ $\{1\}, 2 \leq i \leq 21$.

由于 $\boldsymbol{a}_{1} \notin \boldsymbol{B}$, 将枢轴列 $\boldsymbol{v}_{1}$ 从 $\boldsymbol{V}$ 中剔除, 并加至 $\boldsymbol{W}$ 中成 为 $\boldsymbol{w}_{1}$, 标准化后基向量矩阵如表4所示.

最后, 判断终止条件.

由于 $h=1, M=6$, 故 $h=2$.

又 $\boldsymbol{\theta}=\boldsymbol{V}^{\mathrm{T}} \boldsymbol{a}_{2}=[0,0,0,0,0,0,0,0,0,0,0,0,0,0,0,0$, $0,0,0,-100]^{\mathrm{T}}, \eta=\boldsymbol{W}^{\mathrm{T}} \boldsymbol{a}_{2}=1$. 
表 4 第一次枢轴变换

Table 4 The first pivot transformation

\begin{tabular}{|c|c|c|c|c|c|c|c|c|c|c|c|c|c|c|c|c|c|c|c|c|c|}
\hline 列号 & $w_{1}$ & $\boldsymbol{v}_{1}$ & $v_{2}$ & $v_{3}$ & $v_{4}$ & $v_{5}$ & $\boldsymbol{v}_{6}$ & $v_{7}$ & $v_{8}$ & $\boldsymbol{v}_{9}$ & $\boldsymbol{v}_{10}$ & $v_{11}$ & $\boldsymbol{v}_{12}$ & $v_{13}$ & $v_{14}$ & $\boldsymbol{v}_{15}$ & $v_{16}$ & $v_{17}$ & $\boldsymbol{v}_{18}$ & $v_{19}$ & $\boldsymbol{v}_{20}$ \\
\hline 1 & -0.1700 & -0.8571 & -0.7143 & -0.5714 & $4-0.4286$ & $5-0.2857$ & $7-0.1429$ & 0 & 0.1429 & 0.2857 & 70.4286 & 0.5714 & 40.7143 & 30.8571 & 1 & 0 & 0 & 0 & 0 & 0 & 8.4978 \\
\hline 2 & 0 & 1 & 0 & 0 & 0 & 0 & 0 & 0 & 0 & 0 & 0 & 0 & 0 & 0 & 0 & 0 & 0 & 0 & 0 & 0 & 0 \\
\hline 3 & 0 & 0 & 1 & 0 & 0 & 0 & 0 & 0 & 0 & 0 & 0 & 0 & 0 & 0 & 0 & 0 & 0 & 0 & 0 & 0 & 0 \\
\hline 4 & 0 & 0 & 0 & 1 & 0 & 0 & 0 & 0 & 0 & 0 & 0 & 0 & 0 & 0 & 0 & 0 & 0 & 0 & 0 & 0 & 0 \\
\hline 5 & 0 & 0 & 0 & 0 & 1 & 0 & 0 & 0 & 0 & 0 & 0 & 0 & 0 & 0 & 0 & 0 & 0 & 0 & 0 & 0 & 0 \\
\hline 6 & 0 & 0 & 0 & 0 & 0 & 1 & 0 & 0 & 0 & 0 & 0 & 0 & 0 & 0 & 0 & 0 & 0 & 0 & 0 & 0 & 0 \\
\hline 7 & 0 & 0 & 0 & 0 & 0 & 0 & 1 & 0 & 0 & 0 & 0 & 0 & 0 & 0 & 0 & 0 & 0 & 0 & 0 & 0 & 0 \\
\hline 8 & 0 & 0 & 0 & 0 & 0 & 0 & 0 & 1 & 0 & 0 & 0 & 0 & 0 & 0 & 0 & 0 & 0 & 0 & 0 & 0 & 0 \\
\hline 9 & 0 & 0 & 0 & 0 & 0 & 0 & 0 & 0 & 1 & 0 & 0 & 0 & 0 & 0 & 0 & 0 & 0 & 0 & 0 & 0 & 0 \\
\hline 10 & 0 & 0 & 0 & 0 & 0 & 0 & 0 & 0 & 0 & 1 & 0 & 0 & 0 & 0 & 0 & 0 & 0 & 0 & 0 & 0 & 0 \\
\hline 11 & 0 & 0 & 0 & 0 & 0 & 0 & 0 & 0 & 0 & 0 & 1 & 0 & 0 & 0 & 0 & 0 & 0 & 0 & 0 & 0 & 0 \\
\hline 12 & 0 & 0 & 0 & 0 & 0 & 0 & 0 & 0 & 0 & 0 & 0 & 1 & 0 & 0 & 0 & 0 & 0 & 0 & 0 & 0 & 0 \\
\hline 13 & 0 & 0 & 0 & 0 & 0 & 0 & 0 & 0 & 0 & 0 & 0 & 0 & 1 & 0 & 0 & 0 & 0 & 0 & 0 & 0 & 0 \\
\hline 14 & 0 & 0 & 0 & 0 & 0 & 0 & 0 & 0 & 0 & 0 & 0 & 0 & 0 & 1 & 0 & 0 & 0 & 0 & 0 & 0 & 0 \\
\hline 15 & 0 & 0 & 0 & 0 & 0 & 0 & 0 & 0 & 0 & 0 & 0 & 0 & 0 & 0 & 1 & 0 & 0 & 0 & 0 & 0 & 0 \\
\hline 16 & 0 & 0 & 0 & 0 & 0 & 0 & 0 & 0 & 0 & 0 & 0 & 0 & 0 & 0 & 0 & 1 & 0 & 0 & 0 & 0 & 0 \\
\hline 17 & 0 & 0 & 0 & 0 & 0 & 0 & 0 & 0 & 0 & 0 & 0 & 0 & 0 & 0 & 0 & 0 & 1 & 0 & 0 & 0 & 0 \\
\hline 18 & 0 & 0 & 0 & 0 & 0 & 0 & 0 & 0 & 0 & 0 & 0 & 0 & 0 & 0 & 0 & 0 & 0 & 1 & 0 & 0 & 0 \\
\hline 19 & 0 & 0 & 0 & 0 & 0 & 0 & 0 & 0 & 0 & 0 & 0 & 0 & 0 & 0 & 0 & 0 & 0 & 0 & 1 & 0 & 0 \\
\hline 20 & 0 & 0 & 0 & 0 & 0 & 0 & 0 & 0 & 0 & 0 & 0 & 0 & 0 & 0 & 0 & 0 & 0 & 0 & 0 & 1 & 0 \\
\hline 21 & 0 & 0 & 0 & 0 & 0 & 0 & 0 & 0 & 0 & 0 & 0 & 0 & 0 & 0 & 0 & 0 & 0 & 0 & 0 & 0 & 1 \\
\hline
\end{tabular}

进行第二次旋转变换后, 由于 $\theta_{20} \neq 0$, 则枢轴pivot $=20 . I_{i}^{\mathbf{V}}=\{1,2\} \quad(1 \leq i \leq 19), I_{20}^{\mathbf{v}}=\{1\}, I_{1}^{\mathbf{w}}=\{2\}$. 由于 $\boldsymbol{a}_{2} \notin \boldsymbol{B}$, 将枢轴列 $\boldsymbol{v}_{20}$ 从 $\boldsymbol{V}$ 中剔除并加至 $\boldsymbol{W}$ 中为 $\boldsymbol{w}_{2}$, 如表 5 所示.

由于依然存在 $h<M$, 故 $h=3$.

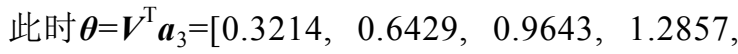
$1.6071,1.9286,2.2500,2.5714,2.8929,3.2143,3.5357$, $3.8571,4.1786,4.5000,4.6500,4.9500,5.2500,5.5500$, $5.8500]^{\mathrm{T}}$, 且 $\boldsymbol{\eta}=\boldsymbol{W}^{\mathrm{T}} \boldsymbol{a}_{3}=[-0.0127,0.0127]^{\mathrm{T}}$.

变换后, 由于 $\boldsymbol{\theta}_{1}=0.3214$ 为非零元素, 将 $h=3$ 添加至 集合 $I_{i}{ }^{\mathbf{v}}(2 \leq i \leq 19)$ 和集合 $I_{j}{ }^{\mathbf{W}}(1 \leq j \leq 2)$ 中, 即 $I_{i} \mathbf{v}=\{1,2,3\}$, $I_{1}^{\mathbf{v}}=\{1,2\}, I_{1}^{\mathbf{w}}=\{2,3\}, I_{2}^{\mathbf{w}}=\{1,3\}$. 又 $\boldsymbol{a}_{3} \in \boldsymbol{B}$, 从 $\boldsymbol{V}$ 中剔除 $v_{1}$ 后得矩阵如表6所示.

依然存在 $h<M$. 令 $h=4$ 后, 有 $\boldsymbol{\theta}=\boldsymbol{V}^{\mathrm{T}} \boldsymbol{a}_{4}=[0,0,0,0,0$, $0,0,0,0,0,0,0,0,0,0,0,0,0]^{\mathrm{T}}, \boldsymbol{\eta}=\boldsymbol{W}^{\mathrm{T}} \boldsymbol{a}_{4}=[0,0]^{\mathrm{T}}$.
由于不存在非零元素, 不执行枢轴变换, 将 $h=4$ 添 加至集合 $I_{i}^{\mathrm{V}}(1 \leq i \leq 18)$ 和集合 $I_{j}^{\mathrm{W}}(1 \leq j \leq 2)$ 中, 即 $I_{i}^{\mathrm{V}}=\{1,2$, $3,4\}, I_{1}^{\mathbf{W}}=\{2,3,4\}, I_{2}^{\mathbf{W}}=\{1,3,4\}$.

继 续令 $h=5$, 则 $\boldsymbol{\theta}=\boldsymbol{V}^{\mathrm{T}} \boldsymbol{a}_{5}=[-0.00001586$, $-0.00001586,-0.00003172,-0.00003172,-0.00004759$, $-0.00004759,-0.00004759,-0.00006345,-0.00006345$, $-0.00007931,-0.00007931,-0.00009517,-0.00009517$, $-1.06676501,-1.20010469,-1.33344437,-1.46678405$, $-1.60012373]^{\mathrm{T}}, \boldsymbol{\eta}=\boldsymbol{W}^{\mathrm{T}} \boldsymbol{a}_{5}=[-0.07931220,0.07931220]^{\mathrm{T}}$.

执行变换后, 由于 $\theta_{1}=0.00001586$ 为非零元素, 将 $h=5$ 添加至集合 $I_{i}^{\mathbf{V}}(2 \leq i \leq 18)$ 和集合 $I_{j}^{\mathbf{W}}(1 \leq j \leq 2)$ 中, 即 $I_{i}^{\mathbf{v}}$ $=\{1,2,3,4,5\}, I_{1}^{\mathbf{v}}=\{1,2,3,4\}, I_{1}^{\mathbf{w}}=\{2,3,4,5\}, I_{2}^{\mathbf{w}}=$ $\{1,3,4,5\}$. 又 $\boldsymbol{a}_{5} \in \boldsymbol{B}$, 从 $\boldsymbol{V}$ 中剔除衴, 得矩阵如表7所示.

由于 $M>5$, 故 $h=6$. 因此, $\boldsymbol{\theta}=\boldsymbol{V}^{\mathrm{T}} \boldsymbol{a}_{6}=[0,0,0,0,0,0,0$, $0,0,0,0,0,0,0,0,0,0,0]^{\mathrm{T}}, \boldsymbol{\eta}=\boldsymbol{W}^{\mathrm{T}} \boldsymbol{a}_{6}=[0,0]^{\mathrm{T}}$. 
由于不存在非零元素, 不执行变换, 将 $h=6$ 添加至 $\quad 5,6\}, I_{1}^{\mathbf{w}}=\{2,3,4,5,6\}, I_{2}^{\mathbf{W}}=\{1,3,4,5,6\}$. 此时, 由于 集合 $I_{i}^{\mathbf{V}}(1 \leq i \leq 17)$ 和集合 $I_{j}^{\mathbf{w}}(1 \leq j \leq 2)$ 中, 即 $I_{i}^{\mathbf{v}}=\{1,2,3,4, \quad h=M=6$, 枢轴变换算法结束, 最终可得应力规范应为

$\boldsymbol{x}=\left[\begin{array}{ccccccccccccccccc}1 & 1 & 2 & 2 & 3 & 4 & 4 & 5 & 5 & 6 & 6 & 7 & -67237 & -75642 & -84047 & -92452 & -100857 \\ -1 & 0 & -1 & 0 & -1 & -2 & -1 & -2 & -1 & -2 & -1 & -2 & 134487 & 151298 & 168109 & 184920 & 201731 \\ -1 & -2 & -2 & -3 & -3 & -3 & -4 & -4 & -5 & -5 & -6 & -6 & -67251 & -75657 & -84063 & -92469 & -100875 \\ 1 & 0 & 0 & 0 & 0 & 0 & 0 & 0 & 0 & 0 & 0 & 0 & 0 & 0 & 0 & 0 & 0 \\ 0 & 1 & 0 & 0 & 0 & 0 & 0 & 0 & 0 & 0 & 0 & 0 & 0 & 0 & 0 & 0 & 0 \\ 0 & 0 & 1 & 0 & 0 & 0 & 0 & 0 & 0 & 0 & 0 & 0 & 0 & 0 & 0 & 0 & 0 \\ 0 & 0 & 0 & 1 & 0 & 0 & 0 & 0 & 0 & 0 & 0 & 0 & 0 & 0 & 0 & 0 & 0 \\ 0 & 0 & 0 & 0 & 1 & 0 & 0 & 0 & 0 & 0 & 0 & 0 & 0 & 0 & 0 & 0 & 0 \\ 0 & 0 & 0 & 0 & 0 & 1 & 0 & 0 & 0 & 0 & 0 & 0 & 0 & 0 & 0 & 0 & 0 \\ 0 & 0 & 0 & 0 & 0 & 0 & 1 & 0 & 0 & 0 & 0 & 0 & 0 & 0 & 0 & 0 & 0 \\ 0 & 0 & 0 & 0 & 0 & 0 & 0 & 1 & 0 & 0 & 0 & 0 & 0 & 0 & 0 & 0 & 0 \\ 0 & 0 & 0 & 0 & 0 & 0 & 0 & 0 & 1 & 0 & 0 & 0 & 0 & 0 & 0 & 0 & 0 \\ 0 & 0 & 0 & 0 & 0 & 0 & 0 & 0 & 0 & 1 & 0 & 0 & 0 & 0 & 0 & 0 & 0 \\ 0 & 0 & 0 & 0 & 0 & 0 & 0 & 0 & 0 & 0 & 1 & 0 & 0 & 0 & 0 & 0 & 0 \\ 0 & 0 & 0 & 0 & 0 & 0 & 0 & 0 & 0 & 0 & 0 & 1 & 0 & 0 & 0 & 0 & 0 \\ 0 & 0 & 0 & 0 & 0 & 0 & 0 & 0 & 0 & 0 & 0 & 0 & 1 & 0 & 0 & 0 & 0 \\ 0 & 0 & 0 & 0 & 0 & 0 & 0 & 0 & 0 & 0 & 0 & 0 & 0 & 1 & 0 & 0 & 0 \\ 0 & 0 & 0 & 0 & 0 & 0 & 0 & 0 & 0 & 0 & 0 & 0 & 0 & 0 & 1 & 0 & 0 \\ 0 & 0 & 0 & 0 & 0 & 0 & 0 & 0 & 0 & 0 & 0 & 0 & 0 & 0 & 0 & 1 & 0 \\ 0 & 0 & 0 & 0 & 0 & 0 & 0 & 0 & 0 & 0 & 0 & 0 & 0 & 0 & 0 & 0 & 1 \\ 0 & 0 & 0 & 0 & 0 & 0 & 0 & 0 & 0 & 0 & 0 & 0 & 0 & 0 & 0 & 0 & 0\end{array}\right]\left[\begin{array}{l}\zeta_{1} \\ \zeta_{2} \\ \zeta_{3} \\ \zeta_{4} \\ \zeta_{5} \\ \zeta_{6} \\ \zeta_{7} \\ \zeta_{8} \\ \zeta_{9} \\ \zeta_{10} \\ \zeta_{11} \\ \zeta_{12} \\ \zeta_{13} \\ \zeta_{14} \\ \zeta_{15} \\ \zeta_{16} \\ \zeta_{17}\end{array}\right]$

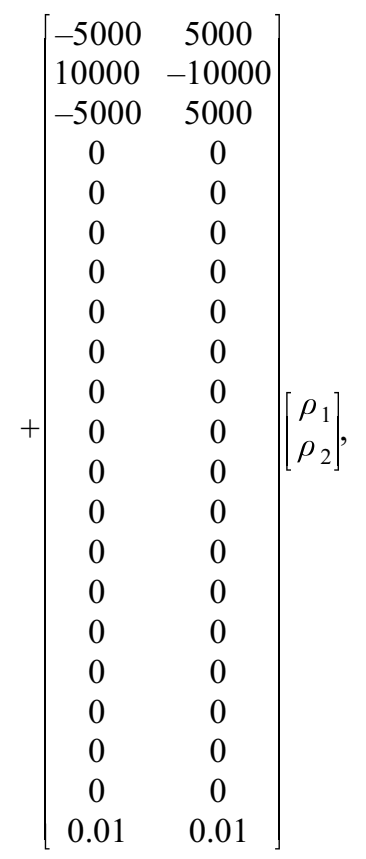

其中, $\zeta_{i}(1 \leq i \leq 17)$ 为任意数, $\rho_{j}(1 \leq j \leq 2)$ 为非负的任意数.

类似地, 若加工要求设定为 $0.5 \mathrm{~mm}$ 时 (即 $\Delta z=250 \times 10^{-3} \mathrm{~mm}$ ), 应力规范可求得为 


$\boldsymbol{x}=\left[\begin{array}{ccccccccccccccccc}1 & 1 & 2 & 2 & 3 & 4 & 4 & 5 & 5 & 6 & 6 & 7 & -67237 & -75642 & -84047 & -92452 & -100857 \\ -1 & 0 & -1 & 0 & -1 & -2 & -1 & -2 & -1 & -2 & -1 & -2 & 134487 & 151298 & 168109 & 184920 & 201731 \\ -1 & -2 & -2 & -3 & -3 & -3 & -4 & -4 & -5 & -5 & -6 & -6 & -67251 & -75657 & -84063 & -92469 & -100875 \\ 1 & 0 & 0 & 0 & 0 & 0 & 0 & 0 & 0 & 0 & 0 & 0 & 0 & 0 & 0 & 0 & 0 \\ 0 & 1 & 0 & 0 & 0 & 0 & 0 & 0 & 0 & 0 & 0 & 0 & 0 & 0 & 0 & 0 & 0 \\ 0 & 0 & 1 & 0 & 0 & 0 & 0 & 0 & 0 & 0 & 0 & 0 & 0 & 0 & 0 & 0 & 0 \\ 0 & 0 & 0 & 1 & 0 & 0 & 0 & 0 & 0 & 0 & 0 & 0 & 0 & 0 & 0 & 0 & 0 \\ 0 & 0 & 0 & 0 & 1 & 0 & 0 & 0 & 0 & 0 & 0 & 0 & 0 & 0 & 0 & 0 & 0 \\ 0 & 0 & 0 & 0 & 0 & 1 & 0 & 0 & 0 & 0 & 0 & 0 & 0 & 0 & 0 & 0 & 0 \\ 0 & 0 & 0 & 0 & 0 & 0 & 1 & 0 & 0 & 0 & 0 & 0 & 0 & 0 & 0 & 0 & 0 \\ 0 & 0 & 0 & 0 & 0 & 0 & 0 & 1 & 0 & 0 & 0 & 0 & 0 & 0 & 0 & 0 & 0 \\ 0 & 0 & 0 & 0 & 0 & 0 & 0 & 0 & 1 & 0 & 0 & 0 & 0 & 0 & 0 & 0 & 0 \\ 0 & 0 & 0 & 0 & 0 & 0 & 0 & 0 & 0 & 1 & 0 & 0 & 0 & 0 & 0 & 0 & 0 \\ 0 & 0 & 0 & 0 & 0 & 0 & 0 & 0 & 0 & 0 & 1 & 0 & 0 & 0 & 0 & 0 & 0 \\ 0 & 0 & 0 & 0 & 0 & 0 & 0 & 0 & 0 & 0 & 0 & 1 & 0 & 0 & 0 & 0 & 0 \\ 0 & 0 & 0 & 0 & 0 & 0 & 0 & 0 & 0 & 0 & 0 & 0 & 1 & 0 & 0 & 0 & 0 \\ 0 & 0 & 0 & 0 & 0 & 0 & 0 & 0 & 0 & 0 & 0 & 0 & 0 & 1 & 0 & 0 & 0 \\ 0 & 0 & 0 & 0 & 0 & 0 & 0 & 0 & 0 & 0 & 0 & 0 & 0 & 0 & 1 & 0 & 0 \\ 0 & 0 & 0 & 0 & 0 & 0 & 0 & 0 & 0 & 0 & 0 & 0 & 0 & 0 & 0 & 1 & 0 \\ 0 & 0 & 0 & 0 & 0 & 0 & 0 & 0 & 0 & 0 & 0 & 0 & 0 & 0 & 0 & 0 & 1 \\ 0 & 0 & 0 & 0 & 0 & 0 & 0 & 0 & 0 & 0 & 0 & 0 & 0 & 0 & 0 & 0 & 0\end{array}\right] \|\left[\begin{array}{c}\zeta_{1} \\ \zeta_{2} \\ \zeta_{3} \\ \zeta_{4} \\ \zeta_{5} \\ \zeta_{6} \\ \zeta_{7} \\ \zeta_{8} \\ \zeta_{9} \\ \zeta_{10} \\ \zeta_{11} \\ \zeta_{12} \\ \zeta_{13} \\ \zeta_{14} \\ \zeta_{15} \\ \zeta_{16} \\ \zeta_{17}\end{array}\right]$

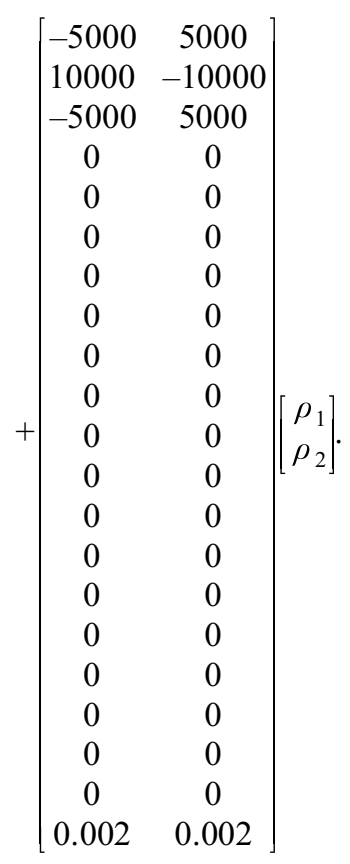

\section{4 残余应力评测}

应力规范即为毛坏出厂的应力标准. 也就是说, 若 毛坏出厂时, 测出的实际应力属于应力规范的解, 则应 力释放造成的变形必然在加工要求范围之内, 否则无 论如何优化加工参数, 加工变形也将超差.

\section{1 评测算法}

假定测出毛坏的当量应力为 $\overline{\boldsymbol{\sigma}}_{X}(z)=\left[\sigma_{1}, \sigma_{2}, \ldots, \sigma_{N-1}\right]^{\mathrm{T}}$, 由于应力规范通解 $\boldsymbol{x}$ 中最后一个应力值 $x_{N}=1$, 那么 $\boldsymbol{X}=$ $\left[\left(\overline{\boldsymbol{\sigma}}_{X}(z)\right)^{\mathrm{T}}, 1\right]^{\mathrm{T}}=\left[\sigma_{1}, \sigma_{2}, \ldots, \sigma_{N-1}, 1\right]^{\mathrm{T}}$. 如果 $\boldsymbol{X}$ 属于式(33)中 $\boldsymbol{x}$ 的解, 则说明毛坏初始残余应力分布合理, 即有应力评 测模型为 
表 5 第二次枢轴变换

Table 5 The second pivot transformation

\begin{tabular}{|c|c|c|c|c|c|c|c|c|c|c|c|c|c|c|c|c|c|c|c|c|c|}
\hline 列号 & $\boldsymbol{w}_{1}$ & $v_{1}$ & $v_{2}$ & $v_{3}$ & $v_{4}$ & $v_{5}$ & $\boldsymbol{v}_{6}$ & $\boldsymbol{v}_{7}$ & $v_{8}$ & $v_{9}$ & $\boldsymbol{v}_{10}$ & $\boldsymbol{v}_{11}$ & $\boldsymbol{v}_{12}$ & $\boldsymbol{v}_{13}$ & $\boldsymbol{v}_{14}$ & $\boldsymbol{v}_{15}$ & $\boldsymbol{v}_{16}$ & $\boldsymbol{v}_{17}$ & $\boldsymbol{v}_{18}$ & $\boldsymbol{v}_{19}$ & $\boldsymbol{w}_{2}$ \\
\hline 1 & -0.0085 & -0.8571 & -0.7143 & -0.5714 & -0.4286 & -0.2857 & -0.1429 & 0 & 0.1429 & 0.2857 & 0.4286 & 0.5714 & 0.7143 & 0.8571 & 1 & 0 & 0 & 0 & 0 & 0 & 0.0850 \\
\hline 2 & 0 & 1 & 0 & 0 & 0 & 0 & 0 & 0 & 0 & 0 & 0 & 0 & 0 & 0 & 0 & 0 & 0 & 0 & 0 & 0 & 0 \\
\hline 3 & 0 & 0 & 1 & 0 & 0 & 0 & 0 & 0 & 0 & 0 & 0 & 0 & 0 & 0 & 0 & 0 & 0 & 0 & 0 & 0 & 0 \\
\hline 4 & 0 & 0 & 0 & 1 & 0 & 0 & 0 & 0 & 0 & 0 & 0 & 0 & 0 & 0 & 0 & 0 & 0 & 0 & 0 & 0 & 0 \\
\hline 5 & 0 & 0 & 0 & 0 & 1 & 0 & 0 & 0 & 0 & 0 & 0 & 0 & 0 & 0 & 0 & 0 & 0 & 0 & 0 & 0 & 0 \\
\hline 6 & 0 & 0 & 0 & 0 & 0 & 1 & 0 & 0 & 0 & 0 & 0 & 0 & 0 & 0 & 0 & 0 & 0 & 0 & 0 & 0 & 0 \\
\hline 7 & 0 & 0 & 0 & 0 & 0 & 0 & 1 & 0 & 0 & 0 & 0 & 0 & 0 & 0 & 0 & 0 & 0 & 0 & 0 & 0 & 0 \\
\hline 8 & 0 & 0 & 0 & 0 & 0 & 0 & 0 & 1 & 0 & 0 & 0 & 0 & 0 & 0 & 0 & 0 & 0 & 0 & 0 & 0 & 0 \\
\hline 9 & 0 & 0 & 0 & 0 & 0 & 0 & 0 & 0 & 1 & 0 & 0 & 0 & 0 & 0 & 0 & 0 & 0 & 0 & 0 & 0 & 0 \\
\hline 10 & 0 & 0 & 0 & 0 & 0 & 0 & 0 & 0 & 0 & 1 & 0 & 0 & 0 & 0 & 0 & 0 & 0 & 0 & 0 & 0 & 0 \\
\hline 11 & 0 & 0 & 0 & 0 & 0 & 0 & 0 & 0 & 0 & 0 & 1 & 0 & 0 & 0 & 0 & 0 & 0 & 0 & 0 & 0 & 0 \\
\hline 12 & 0 & 0 & 0 & 0 & 0 & 0 & 0 & 0 & 0 & 0 & 0 & 1 & 0 & 0 & 0 & 0 & 0 & 0 & 0 & 0 & 0 \\
\hline 13 & 0 & 0 & 0 & 0 & 0 & 0 & 0 & 0 & 0 & 0 & 0 & 0 & 1 & 0 & 0 & 0 & 0 & 0 & 0 & 0 & 0 \\
\hline 14 & 0 & 0 & 0 & 0 & 0 & 0 & 0 & 0 & 0 & 0 & 0 & 0 & 0 & 1 & 0 & 0 & 0 & 0 & 0 & 0 & 0 \\
\hline 15 & 0 & 0 & 0 & 0 & 0 & 0 & 0 & 0 & 0 & 0 & 0 & 0 & 0 & 0 & 1 & 0 & 0 & 0 & 0 & 0 & 0 \\
\hline 16 & 0 & 0 & 0 & 0 & 0 & 0 & 0 & 0 & 0 & 0 & 0 & 0 & 0 & 0 & 0 & 1 & 0 & 0 & 0 & 0 & 0 \\
\hline 17 & 0 & 0 & 0 & 0 & 0 & 0 & 0 & 0 & 0 & 0 & 0 & 0 & 0 & 0 & 0 & 0 & 1 & 0 & 0 & 0 & 0 \\
\hline 18 & 0 & 0 & 0 & 0 & 0 & 0 & 0 & 0 & 0 & 0 & 0 & 0 & 0 & 0 & 0 & 0 & 0 & 1 & 0 & 0 & 0 \\
\hline 19 & 0 & 0 & 0 & 0 & 0 & 0 & 0 & 0 & 0 & 0 & 0 & 0 & 0 & 0 & 0 & 0 & 0 & 0 & 1 & 0 & 0 \\
\hline 20 & 0 & 0 & 0 & 0 & 0 & 0 & 0 & 0 & 0 & 0 & 0 & 0 & 0 & 0 & 0 & 0 & 0 & 0 & 0 & 1 & 0 \\
\hline 21 & 0.0100 & 0 & 0 & 0 & 0 & 0 & 0 & 0 & 0 & 0 & 0 & 0 & 0 & 0 & 0 & 0 & 0 & 0 & 0 & 0 & 0.0100 \\
\hline
\end{tabular}

$$
\boldsymbol{V \rho}+\boldsymbol{W} \boldsymbol{\zeta}=\boldsymbol{X} .
$$

若式(39)中 $\boldsymbol{\zeta}, \boldsymbol{\rho}$ 有解, 则表明 $\boldsymbol{X}$ 为 $\boldsymbol{x}$ 的解.

因为 $\boldsymbol{x}=\boldsymbol{V} \boldsymbol{\zeta}+\boldsymbol{W} \boldsymbol{\rho}$ 中只有 $\boldsymbol{\rho}$ 为非负值, 而 $\boldsymbol{\zeta}$ 为任意值. 令 $\boldsymbol{u}$ 和 $\boldsymbol{v}$ 为非负向量, 即 $\boldsymbol{u} \geq \boldsymbol{0}$ 和 $\boldsymbol{v} \geq \boldsymbol{0}$, 那么任意向量 $\boldsymbol{\zeta}$ 可描 述为

$$
\zeta=\boldsymbol{u}-\boldsymbol{v}
$$

这样, 将式(40)代入式(39)整理后, 式(39)的应力评 测模型等效于下式:

$G y=X$,

s.t.

$\boldsymbol{y} \geq 0$,

其中, $\boldsymbol{G}=[\boldsymbol{V}, \boldsymbol{W},-\boldsymbol{W}], \boldsymbol{y}=\left[\boldsymbol{\rho}^{\mathrm{T}}, \boldsymbol{u}^{\mathrm{T}}, \boldsymbol{v}^{\mathrm{T}}\right]^{\mathrm{T}}$.

式(39)的解的存在性可根据求解下列线性规划问 题进行检验 ${ }^{[17,18]}$, 即 $\max \eta=\boldsymbol{C}^{\mathrm{T}} \boldsymbol{y}$,

s.t.

$\boldsymbol{G} \boldsymbol{y} \leq \boldsymbol{X}$,

$\boldsymbol{y} \geq 0$,

式中, $\boldsymbol{X} \geq \boldsymbol{0}, C_{j}=\sum_{i=1}^{n+1} G_{i, j}$, 且 $C_{j}$ 为 $\boldsymbol{C}$ 的第 $j$ 个元素, 而 $G_{i, j}$ 为 $\boldsymbol{G}$ 的第 $i$ 行第 $j$ 列元素.

这里, 称 $I^{*}=\max (\eta), I=\sum_{i=1}^{n+1} X_{i}$ 分别为理论应力裕 度、实际应力裕度, 则可进一步定义理论应力裕度与 实际应力裕度之差 $\Delta I=I-I^{*}$ 为应力衡度. 那么当且仅 当应力衡度为 0, 即

$\Delta I=0$,

式(39)有解. 此时表明 $\boldsymbol{X}$ 为 $\boldsymbol{x}$ 的解, 也表明测出的实际应 力 $\overline{\boldsymbol{\sigma}}_{X}(z)$ 是合理的, 否则不合理. 
表 6 第三次枢轴变换

Table 6 The three pivot transformation

\begin{tabular}{|c|c|c|c|c|c|c|c|c|c|c|c|c|c|c|c|c|c|c|c|c|}
\hline 列号 & $w_{1}$ & $\boldsymbol{v}_{1}$ & $\boldsymbol{v}_{2}$ & $v_{3}$ & $v_{4}$ & $\boldsymbol{v}_{5}$ & $v_{6}$ & $v_{7}$ & $v_{8}$ & $\boldsymbol{v}_{9}$ & $\boldsymbol{v}_{10}$ & $v_{11}$ & $v_{12}$ & $\boldsymbol{v}_{13}$ & $\boldsymbol{v}_{14}$ & $\boldsymbol{v}_{15}$ & $\boldsymbol{v}_{16}$ & $v_{17}$ & $\boldsymbol{v}_{18}$ & $w_{2}$ \\
\hline 1 & -0.1190 & 1 & 2 & 3 & 4 & 4.9999 & 5.9999 & 6.9999 & 7.9999 & 8.9999 & 9.9999 & 10.9999 & 11.9999 & 12.9999 & 12.3999 & 13.1998 & 13.9998 & 14.7998 & 15.5998 & 0.1190 \\
\hline 2 & 0.0397 & -2 & -3 & & -5 & -6 & -7 & -8 & -9 & -10 & -11 & -12 & -13 & -14 & -14.4666 & -15.3999 & -16.3333 & -17.2666 & $6-18.1999$ & $9-0.0397$ \\
\hline 3 & 0 & 1 & 0 & 0 & 0 & 0 & 0 & 0 & 0 & 0 & 0 & 0 & 0 & 0 & 0 & 0 & 0 & 0 & 0 & 0 \\
\hline 4 & 0 & 0 & 1 & 0 & 0 & 0 & 0 & 0 & 0 & 0 & 0 & 0 & 0 & 0 & 0 & 0 & 0 & 0 & 0 & 0 \\
\hline 5 & 0 & 0 & 0 & 1 & 0 & 0 & 0 & 0 & 0 & 0 & 0 & 0 & 0 & 0 & 0 & 0 & 0 & 0 & 0 & 0 \\
\hline 6 & 0 & 0 & 0 & 0 & 1 & 0 & 0 & 0 & 0 & 0 & 0 & 0 & 0 & 0 & 0 & 0 & 0 & 0 & 0 & 0 \\
\hline 7 & 0 & 0 & 0 & 0 & 0 & 1 & 0 & 0 & 0 & 0 & 0 & 0 & 0 & 0 & 0 & 0 & 0 & 0 & 0 & 0 \\
\hline 8 & 0 & 0 & 0 & 0 & 0 & 0 & 1 & 0 & 0 & 0 & 0 & 0 & 0 & 0 & 0 & 0 & 0 & 0 & 0 & 0 \\
\hline 9 & 0 & 0 & 0 & 0 & 0 & 0 & 0 & 1 & 0 & 0 & 0 & 0 & 0 & 0 & 0 & 0 & 0 & 0 & 0 & 0 \\
\hline 10 & 0 & 0 & 0 & 0 & 0 & 0 & 0 & 0 & 1 & 0 & 0 & 0 & 0 & 0 & 0 & 0 & 0 & 0 & 0 & 0 \\
\hline 11 & 0 & 0 & 0 & 0 & 0 & 0 & 0 & 0 & 0 & 1 & 0 & 0 & 0 & 0 & 0 & 0 & 0 & 0 & 0 & 0 \\
\hline 12 & 0 & 0 & 0 & 0 & 0 & 0 & 0 & 0 & 0 & 0 & 1 & 0 & 0 & 0 & 0 & 0 & 0 & 0 & 0 & 0 \\
\hline 13 & 0 & 0 & 0 & 0 & 0 & 0 & 0 & 0 & 0 & 0 & 0 & 1 & 0 & 0 & 0 & 0 & 0 & 0 & 0 & 0 \\
\hline 14 & 0 & 0 & 0 & 0 & 0 & 0 & 0 & 0 & 0 & 0 & 0 & 0 & 1 & 0 & 0 & 0 & 0 & 0 & 0 & 0 \\
\hline 15 & 0 & 0 & 0 & 0 & 0 & 0 & 0 & 0 & 0 & 0 & 0 & 0 & 0 & 1 & 0 & 0 & 0 & 0 & 0 & 0 \\
\hline 16 & 0 & 0 & 0 & 0 & 0 & 0 & 0 & 0 & 0 & 0 & 0 & 0 & 0 & 0 & 1 & 0 & 0 & 0 & 0 & 0 \\
\hline 17 & 0 & 0 & 0 & 0 & 0 & 0 & 0 & 0 & 0 & 0 & 0 & 0 & 0 & 0 & 0 & 1 & 0 & 0 & 0 & 0 \\
\hline 18 & 0 & 0 & 0 & 0 & 0 & 0 & 0 & 0 & 0 & 0 & 0 & 0 & 0 & 0 & 0 & 0 & 1 & 0 & 0 & 0 \\
\hline 19 & 0 & 0 & 0 & 0 & 0 & 0 & 0 & 0 & 0 & 0 & 0 & 0 & 0 & 0 & 0 & 0 & 0 & 1 & 0 & 0 \\
\hline 20 & 0 & 0 & 0 & 0 & 0 & 0 & 0 & 0 & 0 & 0 & 0 & 0 & 0 & 0 & 0 & 0 & 0 & 0 & 1 & 0 \\
\hline 21 & 0.0100 & 0 & 0 & 0 & 0 & 0 & 0 & 0 & 0 & 0 & 0 & 0 & 0 & 0 & 0 & 0 & 0 & 0 & 0 & 0.0100 \\
\hline
\end{tabular}

\section{2 评测实例}

零件为图8(a)中尺寸为 $200 \mathrm{~mm} \times 20 \mathrm{~mm} \times 4.5 \mathrm{~mm}$ 的 薄板件, 材料为7075-T73铝合金板材. 毛坏尺寸依然为 $200 \mathrm{~mm} \times 20 \mathrm{~mm} \times 6 \mathrm{~mm}$, 图 8 (a)为测出的残余应力分布 曲线.

将曲线离散为 20 层后, 相应的应力值见表 2 , 即测 出的毛坏初始残余应力 $\bar{\sigma}_{X}(z)=[5.81,6.21,6.11,5.16$, $2.71,0.06,-2.44,-5.69,-8.59,-9.34,-9.34,-8.59$, $-5.69,-2.44,0.06,2.71,5.16,6.11,6.21,5.81]^{\mathrm{T}}$. 这样, 引入一个单位应力值后, 对应于 $\bar{\sigma}_{X}(z)$ 的扩展应力 $\boldsymbol{X}=$ $[5.81,6.21,6.11,5.16,2.71,0.06,-2.44,-5.69,-8.59$, $-9.34,-9.34,-8.59,-5.69,-2.44,0.06,2.71,5.16,6.11$, $6.21,5.81,1]^{\mathrm{T}}$.

这里, 薄板件的加工要求假定为 $0.5 \mathrm{~mm}$. 由于式 (37)的应力规范对应的加工要求为 $0.1 \mathrm{~mm}$, 而式(38)对 应于 $0.5 \mathrm{~mm}$ 的加工要求. 为了确保加工变形不超出 $0.5 \mathrm{~mm}$ 的加工要求, 那么扩展应力 $\boldsymbol{X}=[5.81,6.21,6.11$,
$5.16,2.71,0.06,-2.44,-5.69,-8.59,-9.34,-9.34,-8.59$, $-5.69,-2.44,0.06,2.71,5.16,6.11,6.21,5.81,1]^{\mathrm{T}}$ 必为 式(38)的解, 而不是式(37)的解.

由于式(37)和(38)分别给出了各自的向量基矩阵 $\boldsymbol{V}$ 和 $\boldsymbol{W}$, 故根据式(42)和(43)求解出的理论应力裕度和实 际应力裕度见表 8 . 显然, 式(38)的应力衡度为零, 而式 (37)的应力衡度非零. 由此可知, 测出的毛坏初始残余 应力为式(38)的解而非式(37)的解, 表面残余应力 $\bar{\sigma}_{X}(z)$ 引起的加工变形不会超出 $0.5 \mathrm{~mm}$ 的加工要求.

由上节内容可知, 应力 $\bar{\sigma}_{X}(z)$ 产生的加工变形为 $0.22 \mathrm{~mm}$, 大于 $0.1 \mathrm{~mm}$ 而小于 $0.5 \mathrm{~mm}$. 这一事实也验证 了应力规范及应力评测方法的有效性和正确性.

\section{5 衣服件的应力规范与评测}

衣服件为典型的复杂航空整体结构件, 因外廓形 似于衣服, 故名为衣服件. 衣服件属于单面结构, 毛坯 
表 7 第四次枢轴变换

Table 7 The fourth pivot transformation

\begin{tabular}{|c|c|c|c|c|c|c|c|c|c|c|c|c|c|c|c|c|c|c|c|}
\hline 列号 & $\boldsymbol{w}_{1}$ & $v_{1}$ & $v_{2}$ & $v_{3}$ & $v_{4}$ & $v_{5}$ & $v_{6}$ & $\boldsymbol{v}_{7}$ & $v_{8}$ & $v_{9}$ & $\boldsymbol{v}_{10}$ & $\boldsymbol{v}_{11}$ & $v_{12}$ & $v_{13}$ & $\boldsymbol{v}_{14}$ & $\boldsymbol{v}_{15}$ & $\boldsymbol{v}_{16}$ & $v_{17}$ & $\boldsymbol{w}_{2}$ \\
\hline 1 & -5000 & 1 & 1 & 2 & 2 & 3 & 4 & 4 & 5 & 5 & 6 & 6 & 7 & -67237 & -75642 & -84047 & -92452 & -100857 & 5000 \\
\hline 2 & 10000 & -1 & 0 & -1 & 0 & -1 & -2 & -1 & -2 & -1 & -2 & -1 & -2 & 134487 & 151298 & 168109 & 184920 & 201731 & -10000 \\
\hline 3 & -5000 & -1 & -2 & -2 & -3 & -3 & -3 & -4 & -4 & -5 & -5 & -6 & -6 & -67251 & -75657 & -84063 & -92469 & -100875 & 5000 \\
\hline 4 & 0 & 1 & 0 & 0 & 0 & 0 & 0 & 0 & 0 & 0 & 0 & 0 & 0 & 0 & 0 & 0 & 0 & 0 & 0 \\
\hline 5 & 0 & 0 & 1 & 0 & 0 & 0 & 0 & 0 & 0 & 0 & 0 & 0 & 0 & 0 & 0 & 0 & 0 & 0 & 0 \\
\hline 6 & 0 & 0 & 0 & 1 & 0 & 0 & 0 & 0 & 0 & 0 & 0 & 0 & 0 & 0 & 0 & 0 & 0 & 0 & 0 \\
\hline 7 & 0 & 0 & 0 & 0 & 1 & 0 & 0 & 0 & 0 & 0 & 0 & 0 & 0 & 0 & 0 & 0 & 0 & 0 & 0 \\
\hline 8 & 0 & 0 & 0 & 0 & 0 & 1 & 0 & 0 & 0 & 0 & 0 & 0 & 0 & 0 & 0 & 0 & 0 & 0 & 0 \\
\hline 9 & 0 & 0 & 0 & 0 & 0 & 0 & 1 & 0 & 0 & 0 & 0 & 0 & 0 & 0 & 0 & 0 & 0 & 0 & 0 \\
\hline 10 & 0 & 0 & 0 & 0 & 0 & 0 & 0 & 1 & 0 & 0 & 0 & 0 & 0 & 0 & 0 & 0 & 0 & 0 & 0 \\
\hline 11 & 0 & 0 & 0 & 0 & 0 & 0 & 0 & 0 & 1 & 0 & 0 & 0 & 0 & 0 & 0 & 0 & 0 & 0 & 0 \\
\hline 12 & 0 & 0 & 0 & 0 & 0 & 0 & 0 & 0 & 0 & 1 & 0 & 0 & 0 & 0 & 0 & 0 & 0 & 0 & 0 \\
\hline 13 & 0 & 0 & 0 & 0 & 0 & 0 & 0 & 0 & 0 & 0 & 1 & 0 & 0 & 0 & 0 & 0 & 0 & 0 & 0 \\
\hline 14 & 0 & 0 & 0 & 0 & 0 & 0 & 0 & 0 & 0 & 0 & 0 & 1 & 0 & 0 & 0 & 0 & 0 & 0 & 0 \\
\hline 15 & 0 & 0 & 0 & 0 & 0 & 0 & 0 & 0 & 0 & 0 & 0 & 0 & 1 & 0 & 0 & 0 & 0 & 0 & 0 \\
\hline 16 & 0 & 0 & 0 & 0 & 0 & 0 & 0 & 0 & 0 & 0 & 0 & 0 & 0 & 1 & 0 & 0 & 0 & 0 & 0 \\
\hline 17 & 0 & 0 & 0 & 0 & 0 & 0 & 0 & 0 & 0 & 0 & 0 & 0 & 0 & 0 & 1 & 0 & 0 & 0 & 0 \\
\hline 18 & 0 & 0 & 0 & 0 & 0 & 0 & 0 & 0 & 0 & 0 & 0 & 0 & 0 & 0 & 0 & 1 & 0 & 0 & 0 \\
\hline 19 & 0 & 0 & 0 & 0 & 0 & 0 & 0 & 0 & 0 & 0 & 0 & 0 & 0 & 0 & 0 & 0 & 1 & 0 & 0 \\
\hline 20 & 0 & 0 & 0 & 0 & 0 & 0 & 0 & 0 & 0 & 0 & 0 & 0 & 0 & 0 & 0 & 0 & 0 & 1 & 0 \\
\hline 21 & 0.0100 & 0 & 0 & 0 & 0 & 0 & 0 & 0 & 0 & 0 & 0 & 0 & 0 & 0 & 0 & 0 & 0 & 0 & 0.0100 \\
\hline
\end{tabular}

表 8 薄板件的应力衡度

Table 8 Stress balance of thin-walled plate

\begin{tabular}{ccc}
\hline \multirow{2}{*}{ 参数 } & \multicolumn{2}{c}{ 应力规范 } \\
\cline { 2 - 3 } & 加工要求 $0.1 \mathrm{~mm}$ & 加工要求 $0.5 \mathrm{~mm}$ \\
\hline 理论应力裕度 $I^{*}$ & 54.63 & 105.24 \\
实际应力裕度 $I$ & 105.24 & 105.24 \\
应力衡度 $\Delta I$ & 50.61 & 0 \\
\hline
\end{tabular}

选用厚度为 $60 \mathrm{~mm}$ 的7175-T7351铝合金预拉伸板. 7175 -T7351铝合金的弹性模量 $E=71.7 \mathrm{GPa}$, 泊松比 $v=0.33$. 成品的整体结构与尺寸如补充材料中图1所 示, 长2690.5 mm, 宽949.5 mm, 高50 mm.

由于在高速铣削过程中, 腹板底面离毛坏底面距 离为 $4 \mathrm{~mm}$, 故将毛坏沿厚度方向 $Z$ 划分为 19 层, 自下而 上各层的厚度 $\Delta z_{i}(1 \leq i \leq 19)$ 分别为 $4,2,3,3,3,3,3,3,3$,

\section{$3,3,3,3,3,3,3,3,3 \mathrm{~mm}$ 和 $6 \mathrm{~mm}$.}

应力规范求解及应力评测详见补充材料.

\section{6 结论}

(1) 通过分析材料去除导致毛坏内残余应力的释 放, 依据弯曲变形理论推导出残余应力引起的梁类航 空结构件加工变形力学模型. 实验测试结果表明, 力 学模型计算值、有限元仿真值无论是幅值还是变形曲 线, 与实验测量值均具有良好的吻合度.

(2) 通过沿厚向方向逐层施加单位力, 利用有限元 方法计算出各层的变形因子, 提出将加工变形力学模 型分解为变形因子与残余应力之积的单位力法, 实现 加工变形与残余应力的一一对应关系.

(3) 根据加工要求与残余应力的自平衡特性, 建立 了残余应力规范模型. 采用单位变量法和夹逼原则, 通 
过将应力规范模型转化为齐次线性不等式组, 提出求 解规范模型的枢轴变换方法.

(4) 根据实测的扩展应力等于应力规范这一条件, 建立应力评测模型. 通过任意变量转化为任意两个非
负变量之差这一技巧, 建立应力评测模型的解的判定 方法. 只要应力评测模型有解, 表明毛坏内初始残余 应力属于应力规范模型的解, 此时, 零件加工变形必 然满足加工要求.

\section{补充材料}

本文的补充材料见网络版techen.scichina.com. 补充材料为作者提供的原始数据, 作者对其学术质量和内容负责.

\section{参考文献}

1 Sim W. Challenges of residual stresses and part distortion in the civil airframe industry. Int J Microstruct Mater Prop, 2010, 5: 446-455

2 Chantzis D, Van-der-Veen S, J Zettler S, et al. An industrial workflow to minimise part distortion for machining of large monolithic components in aerospace industry. Procedia CIRP, 2013, 8: 281-286

3 Li J, Wang S. Distortion caused by residual stresses in machining aeronautical aluminum alloy parts: Recent advances. Int J Adv Manuf Technol, 2017, 89: 997-1012

4 Koç M, Culp J, Altan T. Prediction of residual stresses in quenched aluminum blocks and their reduction through cold working processes. J Mater Proc Tech, 2006, 174: 342-354

5 Liu L, Sun J, Chen W, et al. Study on the machining distortion of aluminum alloy parts induced by forging residual stresses. Proc Institution Mech Eng Part B-J Eng Manuf, 2017, 231: 618-627

6 Zhang Z, Li L, Yang Y, et al. Machining distortion minimization for the manufacturing of aeronautical structure. Int J Adv Manuf Technol, 2014, 73: $1765-1773$

7 Cerutti X, Mocellin K. Parallel finite element tool to predict distortion induced by initial residual stresses during machining of aeronautical parts. Int J Mater Form, 2015, 8: 255-268

8 Cerutti X, Arsene S, Mocellin K. Prediction of machining quality due to the initial residual stress redistribution of aerospace structural parts made of low-density aluminium alloy rolled plates. Int J Mater Form, 2016, 9: 677-690

9 Nervi S, Szabó B A, Young K A. Prediction of distortion of airframe components made from aluminum plates. AIAA J, 2009, 47: 1635-1641

10 Shang H S. Prediction of the dimensional instability resulting from machining of residually stressed components. Texas Tech University, 1995

11 Liu Q L, Hua J, Jia R Y. Study on the slotting monolithic components under residual stress and its optimization (in Chinese). Mach Design Manuf Eng, 2014, 43: 21-24 [刘秦龙, 华军, 贾瑞艳. 开槽应力释放法优化整体结构件切削加工研究. 机械设计与制造工程, 2014, 43: 21-24]

12 He N, Yang Y F, Li L, et al. Machining deformation of aircraft structure and its control (in Chinese). Aeronautical Manuf Technol, 2009, 328: 3235 [何宁, 杨吟飞, 李亮, 等. 航空结构件加工变形及其控制. 航空制造技术, 2009, 328: 32-35]

13 Huang X, Sun J, Li J. Finite element simulation and experimental investigation on the residual stress-related monolithic component deformation. Int J Adv Manuf Technol, 2015, 77: 1035-1041

14 Sun J, Ke Y L. Study on machining distortion of unitization airframe due to residual stress (in Chinese), J Mech Eng, 2005, 41: 117-122 [孙杰, 柯映林. 残余应力对航空整体结构件加工变形的影响分析. 机械工程学报, 2005, 41: 117-122]

15 Castillo E, Jubete F, Pruneda R E, et al. Obtaining simultaneous solutions of linear subsystems of inequalities and duals. Linear Algebra Appl, 2002, 346: 131-154

16 Richter-Trummer V, Koch D, Witte A, et al. Methodology for prediction of distortion of workpieces manufactured by high speed machining based on an accurate through-the-thickness residual stress determination. Int J Adv Manuf Technol, 2013, 68: 2271-2281

17 Qin G, Zhang W, Wan M. Analysis and optimal design of fixture clamping sequence. J Manuf Sci Eng, 2006, 128: 482-493

18 Qin G, Ye H, Rong Y. A unified point-by-point planning algorithm of machining fixture layout for complex workpiece. Int J Production Res, 2014, 52: 1351-1362

19 Roy U, Liao J, Sun P L, et al. Fixture design synthesis for a flexible manufacturing system. Integr Comp Aid Eng, 1997, 4: 101-113 


\title{
A new approach to deformation control of aeronautical monolithic components of aluminum alloy plates based on stress inverse and stress evaluation
}

\author{
QIN GuoHua ${ }^{1}$, WANG HuaMin ${ }^{2}$, LIN Feng ${ }^{1}$, SUN ShiPing ${ }^{1}$, GUO YiXiang ${ }^{1}$, WU TieJun ${ }^{3} \&$ \\ WU ZhuXi ${ }^{1}$ \\ ${ }^{1}$ School of Aeronautical Manufacturing Engineering, Nanchang Hangkong University, Nanchang 330063, China; \\ ${ }^{2}$ College of Mechanical \& Electrical Engineering, Nanjing University of Aeronautics and Astronautics, Nanjing 210016, China; \\ ${ }^{3}$ Department of Mechanical and Electrical Engineering, Dongguan Polytechnic, Dongguan 523808, China
}

In the high-speed machining process of aeronautical monolithic component, the release of residual stresses is a crucial factor of machining deformations with the material removal of 7075 aluminum alloy thick plates. Therefore, the investigation on the evolution mechanism of machining deformation with residual stress release is crucial for machining quality control. It is vital to achieving a high-efficiency and precise machining process. In this work, the bending deformation theory is employed to formalize the mechanical model of machining deformation, which is influenced by the removal of materials. The finite element method is used to calculate the unit deformations by producing the unit stresses in each layer of the aluminum alloy thick plate. The unit force method is established so that the mechanical model of machining deformation can decompose to be multiple of deformation factor with residual stress. Further, by considering the self-equilibrium of residual stresses, the specification model of residual stresses is proposed based on the fact that the machining requirement represents the control limit of machining deformation. By introducing a new unit variable, the sandwich theorem is employed to convert the specification model into the linear homogeneous inequalities. Therefore, the pivot transformation method is suggested to solve the residual stresses in the specification model. Finally, by representing an arbitrary variable as a difference between two non-negative variables, the linear programming method is proposed to judge whether the measured blank initial residual stress is a solution of stress specification. This can evaluate machining deformation within the machining requirement. The active evaluation of residual stresses, which is deduced from the passive analysis of machining deformations by unit force method and pivot transformation, can provide the proper stress specification for the upper of the machining process. Obviously, the stress specification plays a very important role in the bridge between the blank manufacturing and the machining process.

initial residual stress, high speed machining, machining deformation, linear programming, pivot transformation, stress specification

doi: 10.1360/SST-2019-0089 